Preprint typeset in JHEP style. - HYPER VERSION

SWAT-356

\title{
S-duality of the Leigh-Strassler Deformation via Matrix Models
}

\author{
Nick Dorey, Timothy J. Hollowood and S. Prem Kumar \\ Department of Physics, University of Wales Swansea, Swansea, SA2 8PP, UK \\ E-mail: t.hollowood@swan.ac.uk, n.dorey@swan.ac.uk \\ s.p.kumar@swan.ac.uk
}

\begin{abstract}
We investigate an exactly marginal $\mathcal{N}=1$ supersymmetric deformation of $\mathrm{SU}(N) \mathcal{N}=4$ supersymmetric Yang-Mills theory discovered by Leigh and Strassler. We use a matrix model to compute the exact superpotential for a further massive deformation of the $\mathrm{U}(N)$ Leigh-Strassler theory. We then show how the exact superpotential and eigenvalue spectrum for the $\mathrm{SU}(N)$ theory follows by a process of integrating-in. We find that different vacua are related by an action of the $\mathrm{SL}(2, \mathbb{Z})$ modular group on the bare couplings of the theory extending the action of electric-magnetic duality away from the $\mathcal{N}=4$ theory. We perform non-trivial tests of the matrix model results against semiclassical field theory analysis. We also show that there are interesting points in parameter space where condensates can diverge and vacua disappear. Based on the matrix model results, we propose an exact elliptic superpotential to describe the theory compactified on a circle of finite radius.
\end{abstract}




\section{Introduction}

It has been known for some time that the $\mathcal{N}=4$ supersymmetric Yang-Mills theory in four dimensions possesses exactly marginal deformations. Leigh and Strassler [1] identified two $\mathcal{N}=1$ SUSY-preserving exactly marginal directions other than the $\mathcal{N}=4$ gauge coupling itself, giving rise to a three (complex) dimensional renormalization-group-fixed manifold of $\mathcal{N}=1$ superconformal theories (SCFTs) of which the $\mathcal{N}=4$ fixed line is a subset. Given that strong and weak coupling on the $\mathcal{N}=4$ fixed line are related by the action of $\mathrm{SL}(2, \mathbb{Z})$ on $\tau$, the gauge coupling of the $\mathcal{N}=4$ theory, it is then natural to ask whether this duality extends in some non-trivial way over the entire $\mathcal{N}=1$ fixed manifold.

In this article we answer this question for a particular Leigh-Strassler deformation of the $\mathcal{N}=4$ theory: the so-called " $q$-deformation" $[2]^{1}$. By studying a mass deformation of this Leigh-Strassler SCFT using a recently proposed connection between $\mathcal{N}=1$ theories and matrix models [6-8], we demonstrate that MontonenOlive electric-magnetic duality, extended to the full $\mathrm{SL}(2, \mathbb{Z})$ modular group, extends non-trivially to this space of $\mathcal{N}=1$ superconformal theories accompanied by a welldefined action on the marginal parameters. Not only will we uncover the duality group action in these theories, but we will also provide rather powerful checks on the applicability of the matrix model approach towards solving these $\mathcal{N}=1$ SUSY theories. (Recent checks of the matrix model proposal for other $\mathcal{N}=1$ models have been performed in [3-5]). In particular we will test the superpotential and eigenvalue spectrum obtained from the matrix model against classical field theory results and find nontrivial agreement between the two. Furthermore, rather remarkably the matrix model results for the vacuum expectation values of the effective superpotential can be used to directly infer an exact, dynamical quantum superpotential for the mass deformation of the Leigh-Strassler SCFT compactified on $\mathbb{R}^{3} \times S^{1}$. This superpotential written in terms of the effective fields of the 3 -dimensional theory encodes the entire vacuum structure of the four-dimensional theory and satisfies extremely non-trivial checks to be discussed below. It turns out to be a natural deformation of the elliptic superpotential of [9] for the mass-deformed $\mathcal{N}=4$, or $\mathcal{N}=1^{*}$, theory.

Relevant deformations of SCFTs can tell us a great deal about the CFTs themselves; in particular, as is well-known in the context of $\mathcal{N}=4$ SUSY Yang-Mills and the so-called $\mathcal{N}=2$ elliptic quiver models [8-11,14], the duality properties of the parent theories are inherited by the perturbed theories and manifest themselves as modular properties of various exactly calculable observables in the perturbed theories. In fact, what happens in these known examples is that the holomorphic observables

\footnotetext{
${ }^{1}$ One such deformation has been analysed recently using matrix models in [3].
} 
in one vacuum get mapped into the corresponding observables in a different vacuum (realized in a different phase) under the action of $\mathrm{SL}(2, \mathbb{Z})$ with well-defined modular weights. The holomorphic quantities in question can often be computed exactly and reflect the duality symmetries of the parent theory in this simple fashion.

The lesson to be drawn from this limited set of examples is that the consequences of dualities in these SCFTs are readily visible and more importantly, are readily calculable for certain relevant deformations of these SCFTs. With this in mind, rather than focusing on the SCFT itself we investigate a special mass perturbation of the Leigh-Strassler fixed points. This theory is the $\mathrm{SU}(N)$ gauge theory with $\mathcal{N}=1$ SUSY and three adjoint-valued chiral multiplets $\hat{\Phi}^{+}, \hat{\Phi}^{-}$and $\hat{\Phi}$; the same matter content as the $\mathcal{N}=4$ theory, but with the following classical superpotential ${ }^{2}$

$$
W_{\mathrm{cl}}=\operatorname{Tr}\left(i \lambda \hat{\Phi}\left[\hat{\Phi}^{+}, \hat{\Phi}^{-}\right]_{\beta}+M \hat{\Phi}^{+} \hat{\Phi}^{-}+\mu \hat{\Phi}^{2}\right)
$$

where we have defined the $q$-commutator

$$
\left[\hat{\Phi}^{+}, \hat{\Phi}^{-}\right]_{\beta} \equiv \hat{\Phi}^{+} \hat{\Phi}^{-} e^{i \beta / 2}-\hat{\Phi}^{-} \hat{\Phi}^{+} e^{-i \beta / 2}
$$

and where $\lambda$ and $\beta$ are complex bare couplings. We also introduce the complex bare gauge coupling of this theory $\tau \equiv 4 \pi i / g_{Y M}^{2}+\theta / 2 \pi$. This superpotential represents a deformation away from the $\mathcal{N}=4$ point which is at $\lambda=1, \beta=0$ and $M=\mu=0$. It is worth noting that there are alternative relevant deformations we could have considered involving the operators $\operatorname{Tr} \hat{\Phi}^{+2}$ and $\operatorname{Tr} \hat{\Phi}^{-2}$. These are equivalent to (1.1) as a deformation of the $\mathcal{N}=4$ theory, i.e. when $\beta=0$, but differ once the LeighStrassler marginal deformation is present. In particular, the resulting theories differ in the IR. However, as they only differ by strictly relevant operators they both flow to the same fixed point in the UV: namely the Leigh-Strassler SCFT. The reason for choosing to study the specific relevant deformation (1.1) is simply that the resulting matrix model turns out to be exactly soluble $[6,7,15]$.

Aside from masses for the chiral multiplets $\hat{\Phi}^{ \pm}$and $\hat{\Phi}$, our theory also involves two trilinear deformations, $\mathcal{O}_{1}=\operatorname{Tr} \hat{\Phi}\left[\hat{\Phi}^{+}, \hat{\Phi}^{-}\right]$and $\mathcal{O}_{2}=\operatorname{Tr} \hat{\Phi}\left\{\hat{\Phi}^{+}, \hat{\Phi}^{-}\right\}$. Both the operators $\mathcal{O}_{1}$ and $\mathcal{O}_{2}$ are of course, marginal by power counting but only one of them, or more precisely, only one linear combination of these operators is an exactly marginal deformation of the $\mathcal{N}=4$ theory. Adding $\mathcal{O}_{1}$ to the $\mathcal{N}=4$ Lagrangian only changes the coefficient in front of the $\mathcal{N}=4$ superpotential, but it is an irrelevant operator even at one-loop at the $\mathcal{N}=4$ fixed point [16]. The operator $\mathcal{O}_{1}$ is actually a descendant and not a chiral primary field in the $\mathcal{N}=4$ theory, hence its dimensions

\footnotetext{
${ }^{2}$ We choose a normalization in which the kinetic terms of the chiral multiplets do not have factors of $1 / g_{Y M}^{2}$ in front. In the following, hatted quantities refer to $\mathrm{SU}(N)$ to distinguish them from $\mathrm{U}(N)$.
} 
are not protected. ${ }^{3}$ The second operator $\mathcal{O}_{2}$ on the other hand is known to be exactly marginal at one-loop at the $\mathcal{N}=4$ point [16]. More generally, away from the $\mathcal{N}=4$ line one should expect, for fixed $g_{Y M}$, a particular linear combination of $\mathcal{O}_{1}$ and $\mathcal{O}_{2}$ to be exactly marginal. We have parametrized this particular linear combination via two complex numbers $\lambda$ and $\beta$. In principle, $\lambda$ should be determined as a function of $\beta$ and $g_{Y M}$ on the fixed manifold. Fortunately the specifics of this will not be important for us. Note that in the $\mathrm{SU}(2)$ theory the operator $\mathcal{O}_{2}$ vanishes identically and the above superpotential does not yield a marginal deformation. We will assume that $N>2$ throughout this paper.

The theory with masses for all the fields, as in Eq. (1.1), has a number of vacua, many of which are massive. The canonical examples are the Higgs and confining vacua which will play an important rôle in our discussion. The confining vacua correspond to the trivial classical solution of the $F$-term conditions with $\hat{\Phi}=\hat{\Phi}^{ \pm}=0$ and preserves the full $\mathrm{SU}(N)$ gauge symmetry classically. At low energies the only classically massless fields comprise of the $\mathcal{N}=1$ gauge multiplet which confines and generates a mass gap. There is also a Higgs vacuum where at the classical level the gauge symmetry is completely broken. In addition, there are other massive vacua which are visible classically as solutions that leave a non-abelian gauge subgroup unbroken. Their classification is similar to that of the $\mathcal{N}=1^{*}$ theory $[9,10,18]$. What is interesting about the Leigh-Strassler deformation is that we shall find other vacua which are not present in the $\mathcal{N}=1^{*}$ theory itself. In tandem with this, for special values of the deformation parameter $\beta$, such that $e^{i \beta}$ is a root of unity, vacua can disappear as a result of the condensates diverging.

In this paper, we compute the values of the condensates $\left\langle\operatorname{Tr} \hat{\Phi}^{k}\right\rangle$ and the effective superpotential in all the massive vacua of the $\mathrm{SU}(N)$ theory and we find that Montonen-Olive duality indeed relates the holomorphic condensates in different vacua with a special action on the couplings that we discuss below. This explicit computation is made possible by the recent proposal of Dijkgraaf and Vafa [6] wherein the effective superpotential of the gauge theory is computed by the genus zero free energy of a holomorphic three-matrix integral [7]. The matrix model however, computes the superpotentials for the $\mathrm{U}(N)$ gauge theory. One of our important conclusions is that the $\mathrm{SU}(N)$ superpotential differs non-trivially from its $\mathrm{U}(N)$ counterpart. (See also the work of [5] where a similar issue is discussed). We show however that the former can be unambiguously extracted from the latter by a process of "integrating in" of additional fields present in the $\mathrm{U}(N)$ gauge theory. This difference turns out to be crucial as it is the $\mathrm{SU}(N)$ results that clearly exhibit Montonen-Olive duality while

\footnotetext{
${ }^{3}$ In the context of the AdS/CFT correspondence it is expected that these operators will get mass dimensions $\sim\left(g_{Y M}^{2} N\right)^{1 / 4}[17]$ in the strongly coupled $\mathcal{N}=4$ theory in the large- $N$ limit.
} 
the $\mathrm{U}(N)$ results do not. For the $\mathrm{SU}(N)$ theory we find in a generic $(p, k)$ massive vacuum (up to inconsequential vacuum-independent additive constants)

$$
W_{\mathrm{eff}}^{\mathrm{SU}(N)}=\frac{p N \mu M^{2}}{2 \lambda^{2} \sin \beta} \cdot \frac{\theta_{1}^{\prime}(p \beta / 2 \mid \tilde{\tau})}{\theta_{1}(p \beta / 2 \mid \tilde{\tau})} .
$$

with

$$
\tilde{\tau}=\frac{p \tau_{R}+k}{q} ; \quad \tau_{R} \equiv \tau-\frac{i N}{\pi} \ln \lambda ; \quad p \cdot q=N ; \quad k=0,1, \ldots, q-1 .
$$

The main consequence of this result is that the values of the effective superpotential (and indeed all the eigenvalues of $\hat{\Phi}$ ) in different massive vacua of the theory with deformation parameter $\beta$ are mapped into one another by the action of the $\mathrm{SL}(2, \mathbb{Z})$ transformations on the couplings:

$$
\tau_{R} \longrightarrow \frac{a \tau_{R}+b}{c \tau_{R}+d} ; \quad \beta \longrightarrow \frac{\beta}{c \tau_{R}+d} ; \quad \lambda^{2} \sin \beta \rightarrow \frac{\lambda^{2} \sin \beta}{c \tau_{R}+d},
$$

$a d-b c=1 ; a, b, c, d \in \mathbb{Z}$. In particular, duality of the underlying SCFT is actually realized via modular transformations on a particular combination of the bare couplings $\tau_{R}$ rather than the gauge coupling $\tau$. In addition, $\operatorname{SL}(2, \mathbb{Z})$ permutes vacua of a theory with deformation parameter $\beta$ provided $\beta$ transforms with modular weight -1 as above along with a specific action on $\lambda$ deduced from above.

The vacuum structure and modular transformations described above can also be understood via an exact elliptic superpotential for the theory compactified on $\mathbb{R}^{3} \times S^{1}$. The superpotential is a function of $N$ chiral superfields $X_{a}(a=1,2, \ldots N$, $\left.\sum_{a=1}^{N} X_{a}=0\right)$ which parameterize the Coulomb branch of the compactified theory. These are a complex combination of the Wilson lines and dual photons of the compactified theory. The superpotential

$$
W_{\mathbb{R}^{3} \times S^{1}}=\frac{M^{2} \mu}{2 \lambda^{2} \sin \beta}\left(\frac{\omega_{1}}{\pi}\right) \sum_{a \neq b}\left(\zeta\left(X_{a}-X_{b}+\beta \omega_{1} / \pi\right)-\zeta\left(X_{a}-X_{b}-\beta \omega_{1} / \pi\right)\right)
$$

reproduces the vacua described in Eqs. (1.3) and (1.4), taking the value (1.3) described above. Here, $\zeta(z)$ is the Weierstrass- $\zeta$ function for the torus with half-periods $\omega_{1}=i \pi$ and $\omega_{2}=i \pi \tau_{R}$, i.e. with complex structure $\tau_{R}$. This superpotential also predicts new $\operatorname{SL}(2, \mathbb{Z})$-invariant vacua, not present in the $\mathcal{N}=1^{*}$ theory, whose existence is confirmed by the classical analysis of the four-dimensional field theory.

We also remark that the properties of $\beta$ under modular transformations are similar to those of individual gauge couplings in elliptic quiver theories $[14,19]$. In addition, at non-zero $\beta$ the matrix model solution naturally involves a torus with 
two marked points with $\beta$ parameterizing the separation. These suggest a deeper connection between the Leigh-Strassler and quiver SCFTs.

The layout of this paper is as follows: in Section 2 we show how one can relate the superpotentials for the $\mathrm{U}(N)$ and $\mathrm{SU}(N)$ theories by a process of integrating-in the trace part of the fields. This is very important because the matrix model approach yields the superpotential in the $\mathrm{U}(N)$ theory, whereas, we are primarily interested in the $\mathrm{SU}(N)$ theory. In Section 3, following Dijkgraaf and Vafa, we show how a matrix model can be used to find the superpotential in the confining vacua of the $\mathrm{U}(N)$ theory and therefore by implication in $\mathrm{SU}(N)$ theory. In particular, we find the spectrum of eigenvalues of the adjoint-valued field $\hat{\Phi}$ from which all the holomorphic condensates can be calculated. Section 4 is devoted to an analysis of the vacuum structure of the mass deformed Leigh-Strassler theory from the point-of-view of the tree-level superpotential. In particular, this leads to expressions for the classical limit of the condensates in each of the massive vacua. In addition, for the case of gauge group $\mathrm{SU}(3)$ we find new vacua that are not present in the $\mathcal{N}=1^{*}$ theory. We also show how some of the massive vacua can disappear when the coupling $\beta$ takes particular values. In Section 5 , we return to the matrix model and consider multi-cut solutions that describe all the massive vacua. We then go on to how the structure of vacua can be used to deduce the action of the $\operatorname{SL}(2, \mathbb{Z})$ duality group on the Leigh-Strassler theory itself. The final Section briefly reports on how the results from the matrix model can be used to deduce the exact elliptic superpotential (1.6) for the theory compactified on a circle to three dimensions, generalizing the one for the $\mathcal{N}=1^{*}$ theory constructed in [9].

\section{Relation between the $\mathbf{U}(N)$ and $\mathbf{S U}(N)$ theories}

As we have explained one of our aims is to compute the quantum superpotential and a certain set of holomorphic observables in the massive vacua of the mass deformed $\mathrm{SU}(N)$ Leigh-Strassler SCFT with $\mathcal{N}=1$ SUSY. For this purpose, we would like to employ matrix model techniques that have been proposed recently by Dijkgraaf and Vafa [6]. However, already there is a subtlety. A direct application of the matrix model approach will solve the $\mathrm{U}(N)$ theory since the DV proposal relates the effective superpotentials for $\mathrm{U}(N) \mathcal{N}=1$ gauge theories to the planar diagram expansion of corresponding matrix models. So the first question that we must address is how are the $\mathrm{SU}(N)$ results related to those of the $\mathrm{U}(N)$ gauge theory? We will now show that there is a very specific relation between the effective superpotential of the $\mathrm{SU}(N)$ theory with classical superpotential Eq. (1.1), and the effective superpotential of the $\mathrm{U}(N)$ theory with the same classical superpotential. This relation will eventually 
allow us to extract the $\mathrm{SU}(N)$ results from the matrix model for the $\mathrm{U}(N)$ gauge theory.

\subsection{The $\mathrm{U}(N)$ gauge theory}

The Leigh-Strassler theory with $\mathrm{SU}(N)$ gauge group differs non-trivially from its $\mathrm{U}(N)$ counterpart. The $\mathrm{U}(N)$ theory contains additional neutral chiral multiplets that couple to the chiral superfields transforming in the adjoint representation of the $\mathrm{SU}(N) \subset \mathrm{U}(N)$. These interactions can modify the superpotential and other holomorphic observables of the $\mathrm{SU}(N) \subset \mathrm{U}(N)$ theory. The way this happens is clearly seen from the point-of-view of the $\mathrm{U}(N)$ theory. Let us begin by considering the $\mathrm{U}(N)$ version of the theory, namely, the $\mathcal{N}=1$ SUSY gauge theory with tree level superpotential

$$
W_{\mathrm{cl}}^{\mathrm{U}(N)}=\operatorname{Tr}\left(i \lambda \Phi\left[\Phi^{+}, \Phi^{-}\right]_{\beta}+M \Phi^{+} \Phi^{-}+\mu \Phi^{2}\right),
$$

where $\Phi^{ \pm}$and $\Phi$ are the fields in the adjoint of $U(N)$. Now the fields $\Phi^{ \pm}$and $\Phi$ can be naturally split into their traceless and trace parts:

$$
\Phi \equiv \hat{\Phi}+a ; \quad \Phi^{ \pm} \equiv \hat{\Phi}^{ \pm}+a^{ \pm} ; \quad \operatorname{Tr} \hat{\Phi} \equiv 0 ; \quad \operatorname{Tr} \hat{\Phi}^{ \pm} \equiv 0 .
$$

Here, $\hat{\Phi}^{ \pm}$and $\hat{\Phi}$ are traceless and so transform in the adjoint representation of $\mathrm{SU}(N) \subset \mathrm{U}(N)$ while $a^{ \pm}=\operatorname{Tr} \Phi^{ \pm} / N$ and $a=\operatorname{Tr} \Phi / N$ are neutral. Rewritten in terms of these variables the tree level superpotential (2.1) for the $\mathrm{U}(N)$ theory is

$$
W_{\mathrm{cl}}^{\mathrm{U}(N)}=N\left(M-2 \lambda a \sin \frac{\beta}{2}\right) a^{+} a^{-}+N \mu a^{2}+W_{\mathrm{cl}}^{\mathrm{SU}(N)}\left(a, a^{ \pm}\right)
$$

where

$$
\begin{array}{r}
W_{\mathrm{cl}}^{\mathrm{SU}(N)}\left(a, a^{ \pm}\right)=\operatorname{Tr}\left(i \lambda \hat{\Phi}\left[\hat{\Phi}^{+}, \hat{\Phi}^{-}\right]_{\beta}+\left(M-2 \lambda a \sin \frac{\beta}{2}\right) \hat{\Phi}^{+} \hat{\Phi}^{-}+\mu \hat{\Phi}^{2}\right. \\
\left.-2 \lambda \sin \frac{\beta}{2} a^{-} \hat{\Phi} \hat{\Phi}^{+}-2 \lambda \sin \frac{\beta}{2} a^{+} \hat{\Phi} \hat{\Phi}^{-}\right) .
\end{array}
$$

The main point here is that the neutral trace fields $a$ and $a^{ \pm}$have the effect of modifying the couplings of the $\mathrm{SU}(N)$ fields. For example, the mass $M$ has been renormalized to $M-2 \lambda a \sin \frac{\beta}{2}$. In addition, there are new bilinears in $\hat{\Phi}^{ \pm}$and $\hat{\Phi}$ whose couplings $^{4}$ depend on $a, a^{ \pm}$. (Notice that when $\beta=0$, that is for the $\mathcal{N}=1^{*}$ theory, these modifications disappear and there is no real difference between $\mathrm{SU}(N)$ and

\footnotetext{
${ }^{4}$ Strictly speaking, of course, these "couplings" are actually chiral superfields. But from the point of view the $\mathrm{SU}(N)$ sub-sector of the $\mathrm{U}(N)$ theory, these neutral chiral superfields do appear like couplings that have been elevated to chiral superfields otherwise known as "spurions".
} 
$\mathrm{U}(N)$.) In fact, we show below that because of the symmetries of the theory the VEVs of $a^{+}$and $a^{-}$are forced to be zero self-consistently in the full theory. Thus the only effect of the neutral fields is to modify the mass term $M \rightarrow M^{\prime} \equiv M-2 a \lambda \sin \frac{\beta}{2}$. One might then suspect that answers for pure $\mathrm{SU}(N)$ theory of Eq. (1.1) may be obtained by simply rescaling the $\mathrm{U}(N)$ results by appropriate powers of $M^{\prime}=M-2 a \lambda \sin \frac{\beta}{2}$. We will now see that this is almost correct.

First of all, the fields $a, a^{ \pm}$are blind to all gauge interactions while $\hat{\Phi}, \hat{\Phi}^{ \pm}$experience only $\mathrm{SU}(N)$ gauge interactions, the remaining $\mathrm{U}(1)$ gauge multiplet being decoupled from everything else. Hence in a vacuum where the $\mathrm{SU}(N)$ fields are rendered massive and the gauge interactions generate an effective superpotential for that sector of the theory, we may readily write the effective superpotential for the $\mathrm{U}(N)$ gauge theory as

$$
W_{\mathrm{eff}}^{\mathrm{U}(N)}=N\left(M-2 \lambda a \sin \frac{\beta}{2}\right) a^{+} a^{-}+N \mu a^{2}+W_{\mathrm{eff}}^{\mathrm{SU}(N)}\left(a, a^{ \pm}\right) .
$$

Note that the chiral fields $a, a^{ \pm}$appear as parameters or couplings for the $\mathrm{SU}(N)$ sub-sector, however they are actually dynamical variables in the full theory and their values must be determined by extremizing the full effective superpotential. This procedure can actually be implemented formally by first noting that the $\mathrm{SU}(N)$ sub-sector, from Eq. (2.4), has certain abelian symmetries. Let us define for the sake of convenience $M^{\prime} \equiv M-2 \lambda a \sin \frac{\beta}{2}$. It is then sufficient to consider the following discrete symmetries: (i) $\left(\hat{\Phi}^{ \pm}, a^{ \pm}\right) \rightarrow-\left(\hat{\Phi}^{ \pm}, a^{ \pm}\right)$; (ii) $\left(\hat{\Phi}^{+}, \hat{\Phi}, a^{+}, M^{\prime}, \mu\right) \rightarrow$ $\left(-i \hat{\Phi}^{+}, i \hat{\Phi},-i a^{+}, i M^{\prime},-\mu\right)$; and (iii) $\left(\hat{\Phi}^{-}, \hat{\Phi}, a^{-}, M^{\prime}, \mu\right) \rightarrow\left(-i \hat{\Phi}^{-}, i \hat{\Phi},-i a^{-}, i M^{\prime},-\mu\right)$. The only possible form for the $\mathrm{SU}(N)$ effective superpotential, consistent with these symmetries, analytic in the parameters and which has mass dimension three, is

$$
W_{\mathrm{eff}}^{\mathrm{SU}(N)}=\mu M^{\prime 2} F\left(a^{+} a^{-} / \mu M^{\prime}\right)
$$

for some unknown function $F$. Plugging this back into Eq. (2.5) for the effective $\mathrm{U}(N)$ superpotential, and imposing the $F$-term conditions by extremizing with respect $a, a^{ \pm}$, the only solution that generates a nontrivial effective superpotential is the one where

$$
\left\langle a^{ \pm}\right\rangle=0 ; \quad \text { and } \quad \frac{N M \mu}{2 \lambda \sin \frac{\beta}{2}}\langle a\rangle=W_{\mathrm{eff}}^{\mathrm{U}(N)}
$$

This tells us that in a vacuum of the $\mathrm{U}(N)$ gauge theory, the trace of the adjoint scalar $\Phi$ must be related to the value of the effective superpotential of the $\mathrm{U}(N)$ theory in that vacuum, precisely according to the above equation. This already constitutes a non-trivial prediction for the Dijkgraaf-Vafa approach for solving the $\mathrm{U}(N)$ gauge theory, one that our results from the matrix model must satisfy. 


\subsection{The relation between the $\mathrm{U}(N)$ and $\mathrm{SU}(N)$ superpotentials}

The symmetry arguments above also imply that the effective superpotential of the $\mathrm{SU}(N)$ theory with a classical action as in Eq. (1.1) must be

$$
W_{\mathrm{eff}}^{\mathrm{SU}(N)}=\mu M^{2} F(0) .
$$

But we can easily determine the unknown function $F$ in terms of the $\mathrm{U}(N)$ superpotential using Eqs. (2.5), (2.6) and (2.7) and we find:

$$
W_{\mathrm{eff}}^{\mathrm{SU}(N)}=\frac{W_{\mathrm{eff}}^{\mathrm{U}(N)}}{1-\frac{4 \lambda^{2} \sin ^{2} \beta / 2}{N \mu M^{2}} W_{\mathrm{eff}}^{\mathrm{U}(N)}} .
$$

Anticipating the matrix model results of the following section, we will write this in a slightly different way that may turn out to be more illuminating:

$$
W_{\mathrm{eff}}^{\mathrm{SU}(N)}=\frac{M^{2}}{M^{\prime 2}(\langle a\rangle)}\left[-W_{\mathrm{eff}}^{\mathrm{U}(N)}+\frac{N \mu M^{2}}{4 \lambda^{2} \sin ^{2} \frac{\beta}{2}}\right]-\frac{N \mu M^{2}}{4 \lambda^{2} \sin ^{2} \frac{\beta}{2}} .
$$

In this form, it is apparent that the effective superpotentials of the $\mathrm{U}(N)$ and $\mathrm{SU}(N)$ gauge theories are indeed related by the replacement $M^{\prime}(\langle a\rangle) \rightarrow M$ after subtracting off certain additive constants. This form of the relation will turn out to be quite suggestive and useful when we discuss the matrix model results. Importantly, this simple relation tells us how to extract the $\mathrm{SU}(N)$ answer from the $\mathrm{U}(N)$ result which the matrix model naturally computes.

\section{The $\mathbf{U}(N)$ theory from the matrix model}

According to the Dijkgraaf-Vafa proposal [6], in any given vacuum, the effective superpotential of the $\mathrm{U}(N) \mathcal{N}=1$ gauge theory with classical superpotential Eq. (1.1) is computed in terms of the planar diagram expansion of the three-matrix model partition function expanded around that vacuum

$$
Z=\int\left[d \Phi^{+}\right]\left[d \Phi^{-}\right][d \Phi] \exp -g_{s}^{-1} \operatorname{Tr}\left(i \lambda \Phi\left[\Phi^{+}, \Phi^{-}\right]_{\beta}+M \Phi^{+} \Phi^{-}+\mu \Phi^{2}\right) .
$$

We use the same notation for the matrix fields and the associated superfields in the $\mathrm{U}(N)$ theory. In the matrix model, unlike the field theory, one takes $\Phi^{+}=\left(\Phi^{-}\right)^{\dagger}$

with the fluctuations of $\Phi$ around the saddle point to be Hermitian. This matrix model has been actually solved in a different context [15] in the large- $N$ limit. First one integrates out $\Phi^{ \pm}$and performs a field rescaling $\Phi \rightarrow \Phi / \lambda$ to get

$$
Z=\lambda^{-N^{2}} \int[d \Phi] \frac{\exp -g_{s}^{-1} \mu \operatorname{Tr} \Phi^{2} / \lambda^{2}}{\left|\operatorname{det}\left(M \mathbf{1} \otimes \mathbf{1}-i e^{-i \beta / 2} \Phi \otimes \mathbf{1}+i e^{i \beta / 2} \mathbf{1} \otimes \Phi\right)\right|} .
$$


Now we can follow [15] to obtain the saddle-point equation in the large- $N$ limit. For completeness we will now follow the steps required.

Let $\left\{\phi_{i}\right\}$ denote the eigenvalues of the matrix $\Phi$. Changing the integration variables in the matrix integral by going to the eigenvalue basis introduces a Jacobian: the famous Van der Monde determinant which leads to a repulsive force between the eigenvalues. The second step is a variable change that will eventually yield a simplified form for the saddle-point equation:

$$
\phi_{i}=-M e^{\delta_{i}}+\frac{M}{2 \sin \frac{\beta}{2}} .
$$

In terms of these variables the classical potential for the matrix model eigenvalues takes the following form

$$
\frac{\mu}{\lambda^{2}} \operatorname{Tr} \Phi^{2}=\sum_{i} V\left(\delta_{i}\right)+\frac{\mu N M^{2}}{4 \lambda^{2} \sin ^{2} \frac{\beta}{2}}
$$

where we have defined

$$
V(\delta) \equiv \frac{\mu M^{2}}{\lambda^{2}}\left(e^{2 \delta}-\frac{e^{\delta}}{\sin \frac{\beta}{2}}\right)
$$

In addition to this classical potential, the eigenvalues $\delta_{i}$ also experience pairwise effective interactions induced both by the Van der Monde determinant and the determinant resulting from integrating out $\Phi^{ \pm}$in Eq. (3.2). The eigenvalues $\delta_{i}$ are naturally defined on the complex- $z$ plane with the identification $z \simeq z+2 \pi i$, i.e. a cylinder.

\subsection{Solving the large- $N$ matrix model for the confining vacuum}

In the large- $N$ limit, the eigenvalues form a continuum and condense onto cuts in the complex plane. On can think of these cuts as arising from a quantum smearingout of the classical eigenvalues of $\Phi$. For the confining vacuum all the classical eigenvalues are degenerate, since $\Phi \propto \mathbf{1}$, and so we expect a solution in the matrix model involving a single cut. Multi-cut solutions will be discussed later. The extent of the cut and the matrix model density of eigenvalues $\rho(\delta)$ can be determined from the saddle-point equation in terms of the parameters of the classical potential and the 't Hooft coupling of the matrix model $S=g_{s} N$. The saddle-point equation is most conveniently formulated after defining the resolvent function:

$$
\omega(z)=\frac{1}{2} \int_{a}^{b} d \delta \frac{\rho(\delta)}{\tanh \frac{z-\delta}{2}}, \quad \delta \in[a, b], \quad \int_{a}^{b} \rho(\delta) d \delta=1
$$




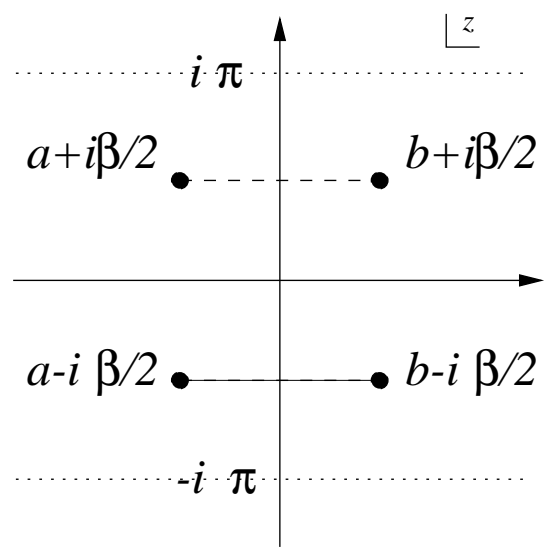

Figure 1: The region over which the function $G(z)$ is defined with its two cuts. The lines $\operatorname{Im} z= \pm i \pi$ are identified.

This function is analytic in $z$ and its only singularity is along a branch cut extending between $[a, b]$. The matrix model spectral density $\rho(\delta)$ is equal to the discontinuity across the cut

$$
\omega(\delta+i \epsilon)-\omega(\delta-i \epsilon)=-2 \pi i \rho(\delta) ; \quad \delta \in[a, b]
$$

The saddle-point equation expresses the zero force condition on a test eigenvalue in the presence of the large- $N$ distribution of eigenvalues along the cut. For the matrix model that we are studying, this condition is best written in terms of the function $G(z)$ defined as

$$
G(z)=U(z)+i S\left(\omega\left(z+i \frac{\beta}{2}\right)-\omega\left(z-i \frac{\beta}{2}\right)\right)
$$

and $\mathrm{U}(z)$ is a polynomial in $e^{z}$ such that

$$
V^{\prime}(z)=-i\left(U\left(z+i \frac{\beta}{2}\right)-U\left(z-i \frac{\beta}{2}\right)\right)
$$

From this definition, one may easily deduce that $G(z)$ is an analytic function on the cylinder and that in the interval $|\operatorname{Im} z| \leq \pi$ it has two cuts $\left[a+i \frac{\beta}{2}, b+i \frac{\beta}{2}\right]$ and $\left[a-i \frac{\beta}{2}, b-i \frac{\beta}{2}\right]$. This is illustrated in Figure 1. In terms of $G(z)$, the matrix model saddle-point equation is

$$
G\left(\delta+i \frac{\beta}{2} \pm i \epsilon\right)=G\left(\delta-i \frac{\beta}{2} \mp i \epsilon\right) ; \quad \delta \in[a, b] .
$$

The saddle-point equations actually provide a gluing condition on the cylinder with two branch cuts. In particular, this defines a torus with two marked points. $G(z)$ is then uniquely specified by gluing condition Eq. (3.10) and asymptotic behaviour at large $z$

$$
\lim _{z \rightarrow \infty} G(z) \rightarrow \frac{\mu M^{2}}{\lambda^{2}}\left[\frac{1}{\sin \beta} e^{2 z}-\frac{1}{2 \sin ^{2} \frac{\beta}{2}} e^{z}+\mathcal{O}\left(e^{-z}\right)\right]
$$



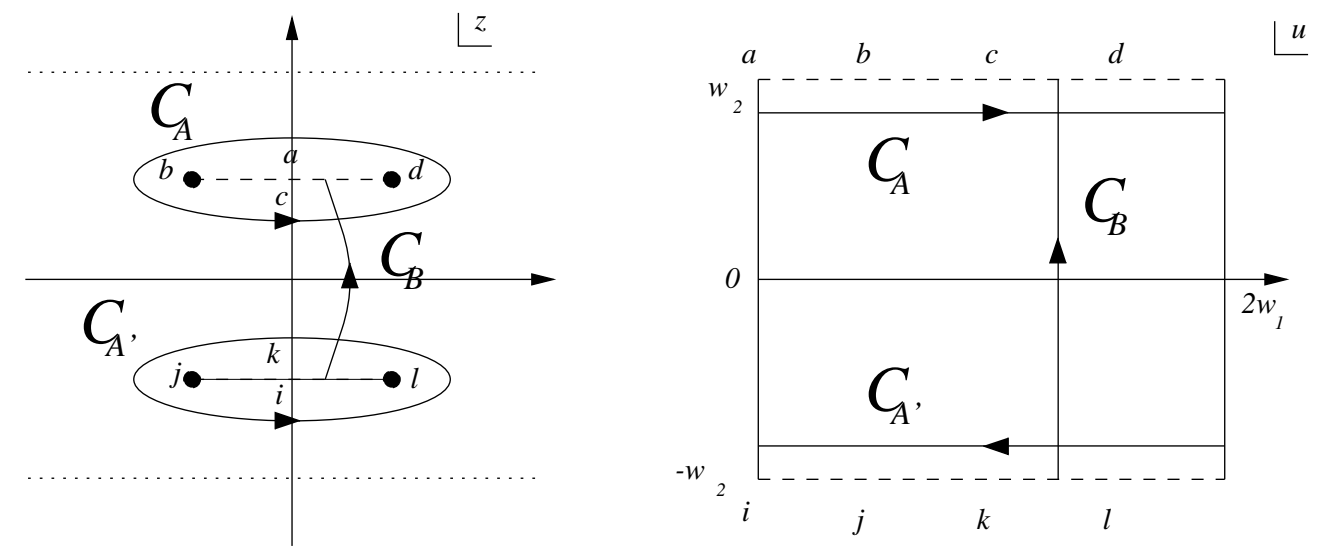

Figure 2: Uniformizing map from the $z$-plane to the torus $E_{\tilde{\tau}}$.

which is a consequence of Eqs. (3.8) and (3.9). With this data we can determine $G(z)$ in a suitable parametrization so that we can then implement the DV proposal and obtain the $\mathrm{U}(N)$ gauge theory superpotential.

\subsection{The $\tilde{\tau}$-torus and the elliptic parametrization}

As we stated earlier, the two-cut cylinder along with the asymptotic properties of $G(z)$ in Eq. (3.11) uniquely specifies a torus $E_{\tilde{\tau}}$ with complex structure parameter $\tilde{\tau}$. As shown in Figure 2, the contour $\mathcal{C}_{A}$ enclosing one of the cuts $\left[a+i \frac{\beta}{2}, b+i \frac{\beta}{2}\right]$ anticlockwise maps to the $A$-cycle of the torus while the contour $\mathcal{C}_{B}$ joining the two cuts maps to the $B$-cycle. We will think of this torus as a quotient of the complex $u$-plane ( $u$ being an auxiliary variable) by a lattice with periods $2 \tilde{\omega}_{1}$ and $2 \tilde{\omega}_{2}$ and with complex structure $\tilde{\tau}=\tilde{\omega}_{2} / \tilde{\omega}_{1}$.

¿From the field theory view-point, the appearance of this torus is not entirely obvious except for the fact that the gauge theory we are studying is connected to the $\mathcal{N}=4$ theory. In the $\mathcal{N}=1^{*}$ case discussed in [7,8], one might have expected the torus to appear due to the relation with the Seiberg-Witten curve of the associated $\mathcal{N}=2^{*}$ theory. For the Leigh-Strassler SCFT the fact that the torus structure is also present is one of the most important conclusions of this paper: as we shall see it leads to an understanding of how $\mathrm{SL}(2, \mathbb{Z})$ acts on the underlying SCFT.

The map $z(u)$ from the $u$-plane to the $z$-plane is specified by the requirements that going around $\mathcal{C}_{A}$ (the $A$-cycle of the torus) returns $z$ to its original value, while traversing the contour $\mathcal{C}_{B}$ (the $B$-cycle of the torus) causes $z$ to jump by an amount $i \beta$, which is the distance between the two cuts in the $z$-plane. Both these operations 
leave $G$ unchanged which is therefore an elliptic function on the $u$-plane. Thus

$$
\begin{array}{lll}
A-\text { cycle }: & z\left(u+2 \tilde{\omega}_{1}\right)=z(u) ; & G\left(z\left(u+2 \tilde{\omega}_{1}\right)\right)=G(z(u)), \\
B \text { - cycle }: & z\left(u+2 \tilde{\omega}_{2}\right)=z(u)+i \beta ; & G\left(z\left(u+2 \tilde{\omega}_{2}\right)\right)=G(z(u)) .
\end{array}
$$

This determines the following unique map $z(u)$ from the $u$-plane to the two-cut cylinder:

$$
\exp z(u)=\frac{1}{2 \tan \frac{\beta}{2}} \cdot \frac{\theta_{1}^{\prime}(0 \mid \tilde{\tau}) \theta_{1}\left(\pi u / 2 \tilde{\omega}_{1}-\beta / 4 \mid \tilde{\tau}\right)}{\theta_{1}^{\prime}(\beta / 2 \mid \tilde{\tau}) \theta_{1}\left(\pi u / 2 \tilde{\omega}_{1}+\beta / 4 \mid \tilde{\tau}\right)}
$$

The fact that $G(z(u))$ is an elliptic function on the $u$-plane along with its asymptotic behaviour at large $z$ (3.11) (corresponding to a pole in the $u$-plane) leads uniquely to

$$
G(z(u))=\frac{\mu M^{2} \tilde{\omega}_{1}^{2} \cos \frac{\beta}{2}}{4 \pi^{2} \lambda^{2} \sin ^{3} \frac{\beta}{2}} \cdot \frac{\theta_{1}(\beta / 2 \mid \tilde{\tau})^{2}}{\theta_{1}^{\prime}(\beta / 2 \mid \tilde{\tau})^{2}}\left[\wp\left(u+\tilde{\omega}_{1} \beta / 2 \pi\right)-\wp\left(\tilde{\omega}_{1} \beta / \pi\right)\right] .
$$

Here, $\wp(u)$ is the Weierstrass function, an elliptic function defined on $E_{\tilde{\tau}}$, and $\theta_{1}(u \mid \tau)$ is the Jacobi theta function of the first kind (see [20] and Appendix A for more details). As expected, $G(z(u))$ is an elliptic function of $u$ with a second order pole in the $u$-plane; $z(u)$ on the other hand is only quasi-elliptic. For a different choice of the deformation potential the asymptotic behaviour of $G(z)$ will change and $G(z(u))$ will end up being an elliptic function of a different order, but significantly $z(u)$ will remain unchanged. There are two special points on the torus $E_{\tilde{\tau}}$ at $u= \pm \beta \tilde{\omega}_{1} / 2 \pi$ which map to the points $z=\mp \infty$. Having determined $G(z)$ in the elliptic parametrization we can now implement the DV proposal to compute the superpotential in the confining vacuum of the $\mathrm{U}(N)$ gauge theory.

\subsection{Superpotential of the $\mathbf{U}(N)$ theory via Dijkgraaf-Vafa}

According the Dijkgraaf-Vafa proposal, the gluino condensate of the gauge theory gets identified with the 't Hooft coupling $S$ of the matrix model. From Eqs. (3.6) and (3.8), the integral of $G(z)$ around one of its cuts, say $\mathcal{C}_{A}$, is equal to $-2 \pi S$, so we can directly compute the gluino condensate of the $\mathrm{U}(N)$ gauge theory in the elliptic parametrization:

$$
\Pi_{A}=2 \pi i S=-i \int_{A} G(z(u)) \frac{d z(u)}{d u} d u .
$$

The second ingredient required to determine the QFT superpotential is the variation in the genus zero free energy of the matrix model in transporting a test eigenvalue from infinity to the endpoint of the cut. This is obtained by integrating the force on a test eigenvalue from infinity to the endpoint of the cut and can be expressed 
as $\Pi_{B}=-i \int_{B} G(z) d z$. There is a slight subtlety here as there is a "zero-point" energy associated with additive constants in the matrix model potential. First note that there is a multiplicative factor of $\lambda^{-N^{2}}$ in the partition function (3.2) and an additive constant in the classical scalar potential (3.4). Both these appear as $S$-dependent contributions to the matrix model genus zero free energy defined as $\mathcal{F}_{0} / g_{s}^{2}=N^{2} \mathcal{F}_{0} / S^{2}$, so that

$$
\frac{\partial \mathcal{F}_{0}}{\partial S}=\Pi_{B}-2 S \ln \lambda+\frac{\mu M^{2}}{4 \lambda^{2} \sin ^{2} \frac{\beta}{2}} ; \quad \Pi_{B}=-i \int_{B} G(z(u)) \frac{d z(u)}{d u} d u
$$

The effective superpotential of the mass deformation of the $\mathrm{U}(N)$ Leigh-Strassler theory in its confining vacuum is obtained by extremizing the following expression with respect to $S$ :

$$
W_{\mathrm{eff}}^{\mathrm{U}(N)}=N \frac{\partial \mathcal{F}_{0}}{\partial S}-2 \pi i \tau S ; \quad \frac{\partial}{\partial S} W_{\mathrm{eff}}^{\mathrm{U}(N)}=0
$$

where $\tau$ is the bare coupling of the theory. The $S$-independent constant in Eq. (3.16) appears as a vacuum-independent additive constant in the superpotential. On the other hand, the term linear in $S$ in Eq. (3.16) actually has the effect of renormalizing the bare coupling $\tau$ so that

$$
\tau \longrightarrow \tau_{R}=\tau-\frac{i N}{\pi} \ln \lambda
$$

Both $\Pi_{A}$ and $\Pi_{B}$ are easy to evaluate in our elliptic parametrization:

$$
\Pi_{A}=\frac{d h(\tilde{\tau})}{d \tilde{\tau}} ; \quad \Pi_{B}=\tilde{\tau} \frac{d h(\tilde{\tau})}{d \tilde{\tau}}-h(\tilde{\tau}),
$$

where

$$
h(\tilde{\tau})=\frac{\mu M^{2} \cos \frac{\beta}{2}}{4 \lambda^{2} \sin ^{3} \frac{\beta}{2}} \cdot \frac{\theta_{1}(\beta / 2 \mid \tilde{\tau})}{\theta_{1}^{\prime}(\beta / 2 \mid \tilde{\tau})} .
$$

It follows straightforwardly that

$$
\frac{\partial}{\partial S} W_{e f f}^{\mathrm{U}(N)}=0 \quad \Longrightarrow \quad \tilde{\tau}=\frac{\tau_{R}}{N}
$$

so that

$$
W_{\mathrm{eff}}^{\mathrm{U}(N)}=-N h\left(\tau_{R} / N\right)+\frac{N \mu M^{2}}{4 \lambda^{2} \sin ^{2} \frac{\beta}{2}} .
$$

Finally we have, therefore, the quantum superpotential of the $\mathrm{U}(N)$ gauge theory in its confining vacuum,

$$
W_{\mathrm{eff}}^{\mathrm{U}(N)}=-\frac{N \mu M^{2} \cos \frac{\beta}{2}}{4 \lambda^{2} \sin ^{3} \frac{\beta}{2}} \cdot \frac{\theta_{1}\left(\beta / 2 \mid \tau_{R} / N\right)}{\theta_{1}^{\prime}\left(\beta / 2 \mid \tau_{R} / N\right)}+\frac{N \mu M^{2}}{4 \lambda^{2} \sin ^{2} \frac{\beta}{2}},
$$


with $\tau_{R}$ defined in (3.18). This result satisfies the first obvious check: in the $\beta \rightarrow 0$ limit with $\lambda=1$ it reproduces the superpotential of the $\mathcal{N}=1^{*}$ theory. The superpotential in the other $N-1$ confining vacua are obtained by repeated $T$-dualities $\tau_{R} \rightarrow \tau_{R}+1$. Note that the $T$-dualities in question naturally act on $\tau_{R}$ rather than the bare coupling $\tau$. Similarly the result for the superpotential has a modular parameter $\tau_{R} / N$ and so we expect that any modular properties we uncover should naturally be a consequence of the modular group acting on $\tau_{R}$ the "renormalized" coupling.

\subsection{Eigenvalues of $\Phi$ in the $\mathrm{U}(N)$ theory and a test}

One of the main results of our earlier work [7] in the context of $\mathcal{N}=1^{*}$ theory was that the map $z(u)$ from the $u$-plane to the $z$-plane, when evaluated along the $A$-cycle of the $\tilde{\tau}$-torus (which maps to the upper cut on the $z$-plane), precisely yields the eigenvalues of the adjoint scalar $\Phi$ of the gauge theory in its confining vacuum, distributed uniformly along this cycle. ${ }^{5}$ We were able to prove this assertion for a class of deformations of the $\mathcal{N}=4$ theory involving masses for two of the adjoint chiral superfields and arbitrary polynomials in the third adjoint field $\Phi$. We will not pursue this generalized analysis in the present case, but we will present strong evidence that the same relation with the field theory eigenvalues holds under the Leigh-Strassler deformation.

For the Leigh-Strassler theory, we first recall that the $\delta_{i}$ are valued in the $z$-plane and these are in turn related to the eigenvalues of the $\Phi$ matrix through Eq. (3.3). Taking into account the fact that the function $G(z)$ is defined in Eq. (3.8) in terms of the resolvent functions $\omega\left(z \pm i \frac{\beta}{2}\right)$ where the positions of the cuts of the latter have been displaced by $\pm i \frac{\beta}{2}$, we conjecture that the function $-M \exp \left(z(u)-i \frac{\beta}{2}\right) / \lambda$ evaluated along the $A$-cycle gives the eigenvalues of $\Phi$ in the $\mathrm{U}(N)$ theory:

$\phi(x)=\frac{M}{2 \lambda \sin \frac{\beta}{2}}\left[-\cos \frac{\beta}{2} \frac{\theta_{1}^{\prime}\left(0 \mid \tau_{R} / N\right)}{\theta_{1}^{\prime}\left(\beta / 2 \mid \tau_{R} / N\right)} \frac{\theta_{3}\left(\pi x-\beta / 4 \mid \tau_{R} / N\right)}{\theta_{3}\left(\pi x+\beta / 4 \mid \tau_{R} / N\right)}+1\right] ; \quad x \in\left[-\frac{1}{2}, \frac{1}{2}\right]$.

Note that the factor $\lambda$ takes into account the field rescaling prior to Eq. (3.3) and we also incorporate the additive shift of $M /\left(2 \lambda \sin \frac{\beta}{2}\right)$ involved in the variable change in Eq. (3.3). This is of course a continuous distribution of eigenvalues appropriate for the $\mathrm{U}(N)$ theory in the large- $N$ limit. But the finite $N$ answer is obtained by simply replacing the continuous index $x$ by a discrete index. For this to be legitimate, it is essential that the field theory eigenvalues be uniformly distributed in the interval

\footnotetext{
${ }^{5}$ In contrast the matrix model eigenvalues are not distributed uniformly along this cycle signalling the crucial difference between the spectral densities of the two systems. Nevertheless, the matrix model provides a means to extract the field theory distributions in a simple way.
} 
$x \in\left[-\frac{1}{2}, \frac{1}{2}\right]$ as we have indeed pointed out earlier. The difference between finite- $N$ and large- $N$ manifests itself in gauge-invariant observables as vacuum-independent operator mixing ambiguities that vanish in the large- $N$ limit [7].

Our results for the eigenvalues in the confining vacuum of the $\mathrm{U}(N)$ theory satisfy several checks. Firstly in the classical limit $\tau_{R} \rightarrow i \infty$, using the properties of the Jacobi theta functions (see Appendix A), Eq. (3.24) vanishes as expected, since the confining vacuum should correspond to the trivial classical solution. Secondly, in the $\beta \rightarrow 0$ limit with $\lambda=1$ the eigenvalues of $\mathcal{N}=1^{*}$ theory in its confining vacuum reproduce the expressions in $[7,21]$. Two further nontrivial tests involve the computation of $\langle\operatorname{Tr} \Phi\rangle$ and $\left\langle\operatorname{Tr} \Phi^{2}\right\rangle$ in the $\mathrm{U}(N)$ theory using the eigenvalues above. With a large- $N$ distribution, the trace reduces to an integral and we find

$$
\begin{aligned}
& \langle\operatorname{Tr} \Phi\rangle=N \int_{-1 / 2}^{1 / 2} d x \phi(x)=\frac{2 \lambda \sin \frac{\beta}{2}}{\mu M} W_{\mathrm{eff}}^{\mathrm{U}(N)}, \\
& \left\langle\operatorname{Tr} \Phi^{2}\right\rangle=N \int_{-1 / 2}^{1 / 2} d x \phi^{2}(x)=\frac{1}{\mu} W_{\mathrm{eff}}^{\mathrm{U}(N)}
\end{aligned}
$$

where $W_{\text {eff }}^{\mathrm{U}(N)}$ is the expression obtained in Eq. (3.23). We show how to compute the associated integrals explicitly in Appendix B. The first (3.25a) demonstrates that the trace of $\Phi$ calculated using the matrix model results satisfies precisely the relation Eq. (2.7) predicted on general grounds from field theory. The second integral is also exactly in accord with field theory expectations since from our general arguments in Section 2, we have $W_{\text {eff }}^{\mathrm{U}(N)} \propto \mu$ and therefore differentiating with respect to $\mu$ gives $\left\langle\operatorname{Tr} \Phi^{2}\right\rangle=W_{\text {eff }}^{\mathrm{U}(N)} / \mu$ precisely (3.25b).

In summary, the eigenvalues of the adjoint scalar $\Phi$ in the confining vacuum of the mass deformed $\mathrm{U}(N)$ Leigh-Strassler theory at large $N$ are given by Eq. (3.24). The eigenvalues of the $\mathrm{U}(N)$ theory at finite $N$ are obtained by substituting the continuous index $x$ with an appropriate discrete index.

\subsection{The $\mathrm{SU}(N)$ superpotential}

We argued in Section 2 that the superpotentials of the $\mathrm{U}(N)$ and $\mathrm{SU}(N)$ theories differ non-trivially, but are nevertheless simply related by Eq. (2.10). The main point there was that the $\mathrm{U}(N)$ theory allows a non-zero $\langle\operatorname{Tr} \Phi\rangle \equiv N\langle a\rangle \neq 0$ to develop in the confining vacuum of the quantum theory. This leads to what is effectively a renormalization of the mass parameter $M \rightarrow M^{\prime}=M-2\langle a\rangle \lambda \sin \frac{\beta}{2}$. According to the result of our field theory analysis of Section 2, the $\mathrm{SU}(N)$ superpotential may be recovered by a rescaling of the $\mathrm{U}(N)$ result by $M^{2} / M^{\prime 2}$, after subtracting 
off a vacuum independent constant. It is quite interesting to note that precisely the same vacuum independent constant makes an appearance in the $\mathrm{U}(N)$ superpotential (3.23) computed in the Dijkgraaf-Vafa approach.

Using our results for the $\mathrm{U}(N)$ superpotential from the previous section and Eq. (2.10), we thus find that in the confining vacuum the $\mathrm{SU}(N)$ theory develops the superpotential,

$$
W_{\mathrm{eff}}^{\mathrm{SU}(N)}=\frac{N \mu M^{2}}{2 \lambda^{2} \sin \beta} \cdot \frac{\theta_{1}^{\prime}\left(\beta / 2 \mid \tau_{R} / N\right)}{\theta_{1}\left(\beta / 2 \mid \tau_{R} / N\right)}-\frac{N \mu M^{2}}{4 \lambda^{2} \sin \frac{\beta}{2}},
$$

where the renormalized coupling is defined in (3.18). This expression for the $\mathrm{SU}(N)$ superpotential has the correct $\beta \rightarrow 0$ limit in that it reproduces the $\mathcal{N}=1^{*}$ superpotential exactly.

\section{6 $\mathrm{SU}(N)$ eigenvalues and condensates}

The eigenvalues $\hat{\phi}_{i}$ of the adjoint scalar $\hat{\Phi}$ in the confining vacuum of the $\operatorname{SU}(N)$ theory are related to the $\mathrm{U}(N)$ eigenvalues by a simple extension of our arguments in Section 2 as

$$
\hat{\phi}(x)=\frac{M}{M^{\prime}}\left(\phi(x)-\frac{M}{2 \lambda \sin \frac{\beta}{2}}\right)+\frac{M}{2 \lambda \sin \frac{\beta}{2}} .
$$

This can be understood simply as a rescaling of the $\mathrm{U}(N)$ spectrum of eigenvalues by $M / M^{\prime}(\langle a\rangle)$ after subtracting out a constant and is in line with the arguments of Section 2 for extracting the $\mathrm{SU}(N)$ superpotential.

Evaluating this explicitly yields

$$
\hat{\phi}(x)=\frac{M}{2 \lambda}\left[-\frac{\theta_{1}^{\prime}\left(0 \mid \tau_{R} / N\right)}{\theta_{1}\left(\beta / 2 \mid \tau_{R} / N\right)} \frac{\theta_{3}\left(\pi x-\beta / 4 \mid \tau_{R} / N\right)}{\theta_{3}\left(\pi x+\beta / 4 \mid \tau_{R} / N\right)}+\frac{1}{\sin \frac{\beta}{2}}\right] ; \quad x \in\left[-\frac{1}{2}, \frac{1}{2}\right] .
$$

We show in Appendix B that with these eigenvalues $\langle\operatorname{Tr} \hat{\Phi}\rangle=0$ and $\left\langle\operatorname{Tr} \hat{\Phi}^{2}\right\rangle=$ $W_{\text {eff }}^{\mathrm{SU}(N)} / \mu$. Once again we remark that with a continuous label $x$ these are really the eigenvalues of the large- $N$ gauge theory but finite- $N$ results are obtained by simply replacing $x$ with a discrete index.

Now that we have the complete distribution of eigenvalues of $\hat{\Phi}$ in the confining vacuum of the $\operatorname{SU}(N)$ theory, every condensate of the form $\left\langle\operatorname{Tr} \hat{\Phi}^{k}\right\rangle$ is automatically computed via the integrals

$$
\left\langle\operatorname{Tr} \hat{\Phi}^{k}\right\rangle=N \int_{-1 / 2}^{1 / 2} d x \hat{\phi}^{k}(x)
$$


We will not undertake the exercise of computing these but, if desired, they can be computed following the methods in Appendix B.

\subsection{Summary}

In this section we have computed the value of the effective superpotential and the spectrum of eigenvalues of $\hat{\Phi}$ in the confining vacuum of the $\mathrm{SU}(N)$ theory. We did this by obtaining the $\mathrm{U}(N)$ superpotential via a matrix model and subsequently extracting the $\mathrm{SU}(N)$ results by a process of integrating-in ${ }^{6}$ discussed in Section 2 . The results of the computation have satisfied several general field theory checks. In addition, the emergence of a quasi-modular function $\left(\theta_{1}^{\prime} / \theta_{1}\right)$ as the superpotential of the $\mathrm{SU}(N)$ theory is strongly suggestive of Montonen-Olive duality being realized in this theory. (In contrast, the $\mathrm{U}(N)$ superpotential being basically the reciprocal of the $\mathrm{SU}(N)$ result, transforms in a complicated way under the modular group.) In order to establish electric-magnetic duality we need to be able to compute the corresponding quantities in other massive vacua of the theory (most notably the Higgs vacuum which should be $S$-dual to the confining phase). We will address this in Sections 4 and 5 which will be devoted to understanding the classical and quantum vacuum structure respectively.

\section{Vacuum structure of deformed $\mathrm{SU}(N)$ Leigh-Strassler the- ory}

In this section we exhibit the classical vacuum structure of the $\mathrm{SU}(N)$ theory. Importantly these classical configurations will serve as the starting point for analysing other saddle points of the matrix model and thence obtaining the effective quantum superpotential in all the massive vacua. Interestingly the classical solutions (the eigenvalues) for the Higgs vacuum that we find here will be shown to be related by $S$-duality to the quantum eigenvalue spectrum of the confining vacuum that we have already obtained in the previous section.

The vacuum structure of the mass deformed $\mathrm{SU}(N)$ Leigh-Strassler theory turns out to be subtly different from that of the $\mathcal{N}=1^{*}$ theory: there are new vacua and

\footnotetext{
${ }^{6}$ We remark that one might have expected to obtain the $\mathrm{SU}(N)$ superpotential and eigenvalues directly from the matrix model by imposing a constraint on the matrices via a Lagrange multiplier. However this turns out not to work in any simple way, the primary reason being, as pointed out in [7], that the matrix model eigenvalue spectrum is completely different from the field theory spectrum and tracelessness of the latter translates into a rather complicated constraint on the former.
} 
old vacua can disappear. In order to describe the vacuum structure, we start with the tree-level superpotential (1.1). At the classical level vacua are determined by solving the $F$ - and $D$-flatness conditions modulo gauge transformations. As usual, if one is interested in holomorphic information it is sufficient to solve the $F$-flatness conditions modulo complex gauge transformations. These conditions follow from extremizing the tree-level superpotential (1.1]): ${ }^{7}$

$$
\mathbb{P}\left[\hat{\Phi}^{+}, \hat{\Phi}^{-}\right]_{\beta}=-i \frac{2 \mu}{\lambda} \hat{\Phi}, \quad \mathbb{P}\left[\hat{\Phi}, \hat{\Phi}^{+}\right]=-i \frac{M}{\lambda} \hat{\Phi}^{+}, \quad \mathbb{P}\left[\hat{\Phi}^{-}, \hat{\Phi}\right]=-i \frac{M}{\lambda} \hat{\Phi}^{-}
$$

In the above, $\mathbb{P}$ is the projector onto the traceless part which must be imposed since we are dealing with the $\mathrm{SU}(N)$, rather than the $\mathrm{U}(N)$, theory. Complex gauge transformation can be used to diagonalize $\hat{\Phi}$.

In the limit $\beta=0$, the theory becomes the basic $\mathcal{N}=1^{*}$ theory and the conditions (4.1) reduce to the Lie algebra of $\mathrm{SU}(2)$. Let us briefly recall the solutions in that case. Each solution is associated to a representation of $\mathrm{SU}(2)$ of dimension $N$ where $\hat{\Phi}$ and $\hat{\Phi}^{ \pm}$are, up to a trivial re-scaling, $J_{3}$ and $J_{ \pm}$, respectively. Each representation of $\mathrm{SU}(2)$ of dimension $N$ is associated to a partition $N \rightarrow n_{1}+\cdots+n_{q}$, where each integer $n_{i}$ is the dimension of each irreducible component. In general the unbroken gauge group has abelian factors and the phase is Coulomb, or massless. The massive vacua are associated to the the equi-partitions $N=q \cdot p$ for which the unbroken gauge group is $\mathrm{SU}(q)$. In such a vacuum, the low energy description is pure $\mathcal{N}=1$ with gauge group $\mathrm{SU}(q)$ which has $q$ inequivalent quantum vacua. Hence, there are $\sum_{q \mid N} q$ vacua with a mass gap (the sum being over the positive integer divisors of $N$ ).

Now we consider the case when $\beta \neq 0$. Each of the vacua of the $\mathcal{N}=1^{*}$ theory remains a vacuum of the deformed theory, at least for generic values of $\beta$. These deformed solutions have the following form. Firstly, the block form of the solutions associated to the partition $N \rightarrow n_{1}+\cdots+n_{q}$ is preserved. Let us concentrate on one of the blocks of dimension $n$. For $\beta=0, \hat{\Phi}$ and $\hat{\Phi}^{ \pm}$are equal to $J_{3}$ and $J_{ \pm}$, up to trivial re-scaling, of the irreducible $\mathrm{SU}(2)$ representation of dimension $n$ :

$$
\beta=0: \quad \hat{\Phi}=-\frac{M}{\lambda} J_{3} ; \quad \Phi^{ \pm}=\frac{2 \mu M}{\lambda} J_{ \pm} .
$$

When $\beta \neq 0$, the non-zero elements of $\hat{\Phi}^{ \pm}$have the same pattern as $J_{ \pm}$(so only the elements just above and below the diagonal, respectively are non-zero) but the numerical values are changed. The elements of diagonal matrix $\hat{\Phi}$ in the block in

\footnotetext{
${ }^{7}$ Note that the $F$-term equations are rather similar to the " $q$-deformed" $\mathrm{SU}(2)$ algebra. They are similar to, but not the same as the equations encountered in [2]. The authors of [2] considered a slightly different mass deformation and a $\mathrm{U}(N)$ gauge group.
} 
question are found to be

$$
\hat{\phi}_{j}=\frac{M}{2 \lambda}\left(\frac{1}{\sin \frac{\beta}{2}}-\frac{n}{\sin \frac{n \beta}{2}} e^{-i \beta(n+1-2 j) / 2}\right), \quad j=1, \ldots, n .
$$

Notice that when $\beta \rightarrow 0$, $\hat{\Phi}$, in this block, becomes equal to $-(M / \lambda) J_{3}$ as expected.

For generic $\beta$, these deformed vacua are massive/massless according to their type when $\beta=0$. However, for specific values of $\beta$ vacua can migrate off to infinity in field space and hence cease to be genuine vacua of the field theory. This occurs when the eigenvalues of $\hat{\Phi}$ diverge. It is easy to see that this will occur in a block of dimension $n$ when the deformation parameter

$$
\beta=\frac{2 \pi k}{n}, \quad k \in \mathbb{Z} .
$$

In one of the $N / p$ massive vacua with unbroken $\mathrm{SU}(N / p)$ symmetry the eigenvalues of $\hat{\Phi}$ are given by (4.3) with $n=p$ and where each eigenvalue has degeneracy $N / p$. From this spectrum, we can calculate the classical values for the condensates; for instance, for the quadratic condensate:

$$
\left\langle\operatorname{Tr} \hat{\Phi}^{2}\right\rangle=\frac{M^{2} N}{4 \lambda^{2} \sin ^{2} \frac{\beta}{2}}\left(p \frac{\tan \frac{\beta}{2}}{\tan \frac{p \beta}{2}}-1\right) .
$$

Notice that this condensate diverges when $\beta=2 \pi k / p, k \in \mathbb{Z}$ in accord with (4.4).

\subsection{New classical branches}

As well as the original massive vacua, there are also new vacua which are not present when $\beta=0$. We illustrate this is in the simplest case where the gauge group is $\mathrm{SU}(3)$. In this case there is a new vacuum, more precisely a moduli space of vacua, in which $\hat{\Phi}$ and $\hat{\Phi}^{ \pm}$are all, up to complex gauge transformations, diagonal:

$$
\begin{aligned}
\hat{\Phi} & =\frac{M}{2 \lambda \sin \frac{\beta}{2}} \operatorname{diag}\left(x_{1}, x_{2}, x_{3}\right), \\
\hat{\Phi}^{+} & =\frac{\sqrt{\mu M / 2}}{\lambda \sin \frac{\beta}{2}} \rho \operatorname{diag}\left(\left(1-x_{1}\right)^{-1},\left(1-x_{2}\right)^{-1},\left(1-x_{3}\right)^{-1}\right), \\
\hat{\Phi}^{-} & =\frac{\sqrt{\mu M / 2}}{\lambda \sin \frac{\beta}{2}} \xi \operatorname{diag}\left(\left(1-x_{1}\right)^{-1},\left(1-x_{2}\right)^{-1},\left(1-x_{3}\right)^{-1}\right) .
\end{aligned}
$$

Here, $\rho$ and $\xi$ are two complex moduli of the solution and $x_{i}, i=1,2,3$ are the three distinct roots of the cubic

$$
x^{3}-3 x+2-\rho \xi=0 .
$$


Note that the tracelessness of $\hat{\Phi}$ and $\hat{\Phi}^{ \pm}$is guaranteed by the form of the cubic (4.7). Clearly these solutions diverge to infinity when $\beta \rightarrow 0$. However, the moduli space of the solutions also has additional branches which occur whenever

$$
e^{i \beta / 2} x_{i}-e^{-i \beta / 2} x_{j}=2 i \sin \frac{\beta}{2}
$$

In this case, an off-diagonal component of either of $\left(\hat{\Phi}^{ \pm}\right)_{i j}$ can become non-zero. The quadratic condensate in this space of vacua is

$$
\mathrm{SU}(3): \quad\left\langle\operatorname{Tr} \hat{\Phi}^{2}\right\rangle=\frac{3 M^{2}}{2 \lambda^{2} \sin ^{2} \frac{\beta}{2}} .
$$

Clearly this diverges when $\beta=0$. We will see that these new vacua are important for checking a conjecture that we make later.

\section{Electric-magnetic duality in the Leigh-Strassler Deforma- tion}

The question we now address is what becomes of the $S$-duality of the $\mathcal{N}=4$ theory under the Leigh-Strassler deformation? Specifically we are considering a deformation of the $\mathcal{N}=4$ Lagrangian of the form;

$$
\mathcal{L}=\mathcal{L}_{\mathcal{N}=4}+\int d^{2} \theta \Delta W
$$

where the deforming superpotential can be written as

$$
\Delta W=i\left(\lambda \cos \frac{\beta}{2}-1\right) \mathcal{O}_{1}-\lambda \sin \frac{\beta}{2} \mathcal{O}_{2}+M \operatorname{Tr} \hat{\Phi}^{+} \hat{\Phi}^{-}+\mu \operatorname{Tr} \hat{\Phi}^{2}
$$

where, as in Section $1, \mathcal{O}_{1}=\operatorname{Tr} \hat{\Phi}\left[\hat{\Phi}^{+}, \hat{\Phi}^{-}\right]$and $\mathcal{O}_{2}=\operatorname{Tr} \hat{\Phi}\left\{\hat{\Phi}^{+}, \hat{\Phi}^{-}\right\}$. At the $\mathcal{N}=4$ point, the operators appearing in the deforming superpotential transform under $S$ duality in a definite way. Adding these operators to the superpotential therefore breaks modular invariance. However, as is standard when dealing with broken symmetries, we should be able to restore invariance by allowing the corresponding couplings to transform in an appropriate way. This strategy was implemented in detail for the $\mathcal{N}=1^{*}$ case $\beta=0$ in [13]. We will now review this briefly and extend this discussion to the marginal deformation.

Using the conventions described in $[13],{ }^{8}$ the superspace measure $d^{2} \theta$ has holomorphic modular weight -2 . Hence for modular invariance of the Lagrangian, the

\footnotetext{
${ }^{8}$ See the final paragraph of Section 3 of this reference.
} 
superpotential must have weight +2 . In the $\mathcal{N}=4$ theory, the mass operators $\operatorname{Tr} \hat{\Phi}^{+} \hat{\Phi}^{-}$and $\operatorname{Tr} \hat{\Phi}^{2}$ are both part of the multiplet of chiral primary operators which transform as second rank symmetric traceless tensors under the $\mathrm{SO}(6)$ R-symmetry. Their modular weights can be deduced via the AdS/CFT correspondence by comparison with the dual fields in IIB supergravity [12]. In fact they have holomorphic modular weight +2 and the Lagrangian will be invariant if we assign the mass parameters $M$ and $\mu$ weight zero. Although there is no explicit breaking of modular invariance, it is important to remember that the theory has various vacuum states in which these mass operators acquire expectation values, thereby 'spontaneously' breaking $S$-duality. In other words, modular transformations map the observables in one vacuum to those in another. Indeed, this property is manifest in the exact results of $[11,13]$.

Similar arguments should apply to the Leigh-Strassler theory at least near the $\mathcal{N}=4$ point where the modular properties of the deforming operators are known. If we set $\lambda=1$ and work to linear order in $\beta$, the deformation only contains the cubic operator $\mathcal{O}_{2}$. This operator is also part of an $\mathcal{N}=4$ chiral primary multiplet consisting, in this case, of third rank symmetric traceless tensors of $S O(6)$. In the conventions of [13] it has modular weight +3 . To restore invariance under $S$-duality we should therefore assign the parameter $\beta$ holomorphic modular weight -1 . In Section 5.2, we will find the appropriate generalization of this transformation property throughout the parameter space, by using the matrix model to find the exact superpotential in each of the massive vacua. In Section 5.1 we see what can be deduced by looking at the Higgs and confining vacua alone.

\subsection{A first look at $S$-duality}

Let us concentrate on the Higgs and confining vacua. In the $\mathcal{N}=1^{*}$ limit, it is well known that each observable in the Higgs vacuum is simply related by $S$-duality, $\tau \rightarrow-1 / \tau$, to the corresponding observable in the confining vacuum. This is an explicit realization of the Montonen-Olive electric-magnetic duality of the $\mathcal{N}=4$ theory. The question is whether a similar duality persists under the Leigh-Strassler deformation.

If $S$-duality is realized in this theory it should exchange the Higgs and confining vacua. Since we already have the eigenvalues in the confining vacuum of the $\mathrm{SU}(N)$ theory a simple and powerful test emerges: use $S$-duality to deduce the eigenvalues in the Higgs vacuum and then take the classical limit and compare with the spectrum (4.3) $(n=N)$ deduced from the $F$-flatness conditions. In the next section, we shall use the matrix model to solve for the superpotential in each of the massive vacua 
and this uncovers the action of the whole of the modular group.

The eigenvalues of $\hat{\Phi}$ in the confining vacuum are given in (3.28). (Recall that the eigenvalues in this vacuum have the property that they vanish in the classical limit.) The first thing to notice is that the modular parameter of the elliptic function parametrizing the eigenvalue spectrum is $\tau_{R} / N$ with $\tau_{R}=\tau-(i N / \pi) \ln \lambda$, where $\tau$ is the gauge coupling. Hence $S$-duality, if realized, must act on the renormalized coupling $\tau_{R}$ as $\tau_{R} \rightarrow-1 / \tau_{R}$ rather than $\tau$ itself. Under this action, the modular parameter of the elliptic functions changes as $\tau_{R} / N \rightarrow-1 /\left(\tau_{R} N\right)$. But now we want to take the classical limit $\tau_{R} \rightarrow i \infty$ and this can be done easily after performing a modular transformation $-1 /\left(\tau_{R} N\right) \rightarrow \tau_{R} N$ on the (quasi-)elliptic functions. Using the modular transformation properties of the theta functions (see Appendix A) we find

$$
\hat{\phi}(x) \longrightarrow-\tau_{R} \frac{N M}{2 \lambda \sin \frac{N \tau_{R} \beta}{2}} e^{-i N \tau_{R} \beta x}+\frac{M}{2 \lambda \sin \frac{\beta}{2}} ; \quad x \in\left[-\frac{1}{2}, \frac{1}{2}\right] .
$$

Apart form the additive constant, this coincides precisely with the expression Eq. (4.3) $(n=N)$ for the eigenvalues in the Higgs vacuum of the deformed $\mathrm{SU}(N)$ LeighStrassler theory with $\beta$ replaced by $\tau_{R} \beta$. Equivalently, the Higgs and confining vacua of the theory with deformation parameter $\beta$ are simply exchanged under the combined operation $\tau_{R} \rightarrow-1 / \tau_{R}$ and $\beta \rightarrow \beta / \tau_{R}$

The overall factor of $\tau_{R}$ in the above expression is expected because the eigenvalues have modular weight one. The fact that the additive constants are different is to be expected: even in the context of $\mathcal{N}=1^{*}$ theory the matrix model approach only yields results that are $S$-duality covariant up to a vacuum independent shift [7]. This additive shift will be discussed in more detail in the next section. We should also point out that after $S$-duality on the spectrum of eigenvalues in the confining vacuum we have taken a classical limit above. In this limit, the exact transformation properties of the couplings $\lambda, \beta$ are not visible. These will become clear in the next section.

The results of this simple analysis are that there is indeed a form of $S$-duality that relates the Higgs and confining vacua of the theory with deformation parameter $\beta$. In particular this duality acts on the bare couplings of the Leigh-Strassler theory as

$$
\tau_{R} \longrightarrow-1 / \tau_{R}, \quad \beta \longrightarrow \beta / \tau_{R}
$$

This is consistent with $\beta$ having modular weight -1 as expected. Recall that the bare theory has 3 couplings $\tau, \lambda$ and $\beta$ with one non-trivial relation, so that (5.4) determines the action of $S$-duality on the bare theory up to the unknown relation. In the next section we expand the discussion of duality to include the whole of the modular group $\mathrm{SL}(2, \mathbb{Z})$ and deduce the action on all three of the bare couplings. 


\subsection{Multi-cut solutions of the matrix model and $\mathrm{SL}(2, \mathbb{Z})$}

Now that we have described the structure of massive vacua of the Leigh-Strassler deformed theory, we can return to the matrix model and ask whether we can solve for the exact superpotential and condensates in each of these vacua. This will turn out to be very important because it will prove that there is an action of electricmagnetic duality on the Leigh-Strassler deformation of the $\mathcal{N}=4$ theory. In fact this extends to the action of the full $\mathrm{SL}(2, \mathbb{Z})$ modular group.

The way to use the matrix model to solve for the theory in an arbitrary massive vacuum is described for the $\mathcal{N}=1^{*}$ theory in [8]. One finds that the same techniques can be used in the deformed matrix model described in Section 3. In order to describe the massive vacua with unbroken $\mathrm{SU}(N / p)$ symmetry, one needs a multi-cut solution of the matrix model. Of course the matrix model computes the superpotential in the $\mathrm{U}(N)$ theory and we then use (2.9) to deduce it in the $\mathrm{SU}(N)$ theory.

We start with the observation that the classical eigenvalues in the massive vacua with unbroken $\mathrm{SU}(N / p)$ are given by (4.3) with $n=p$, each with degeneracy $N / p$. The only thing that will be important for us will be the range of the phases of these classical eigenvalues. The classical eigenvalues of the $\mathrm{U}(N)$ gauge theory also have exactly the same phases in the corresponding massive vacua. As we have seen explicitly in the case of the confining vacuum, the eigenvalues of the field theory are essentially proportional to $\exp [z]$. This directs us to consider a $p$-cut solution of the matrix model with the cuts in the $z$-plane situated at

$$
[a+i \beta(p+1-2 j) / 2, b+i \beta(p+1-2 j) / 2] ; \quad j=1, \ldots, p
$$

where these positions are dictated precisely by the phases of the classical eigenvalues. The classical eigenvalues are clustered in groups of $N / p$ at each of these points, but the pairwise effective interactions induced in the matrix model will cause each cluster to spread out into a cut.

Following [8], we then make an ansatz where the cuts have the same length and the same filling fractions. It can then be shown that $G(z)$ defined in (3.8) again only has two cuts, as in the confining vacuum, but where the cuts are shifted to $\pm[a+i \beta p / 2, b+i \beta p / 2]$. This modifies the map $z(u)$ in (3.13) by a shift $\beta \rightarrow p \beta$ :

$$
\exp z(u)=\frac{1}{2 \tan \frac{p \beta}{2}} \cdot \frac{\theta_{1}^{\prime}(0 \mid \tilde{\tau}) \theta_{1}\left(\pi u / 2 \tilde{\omega}_{1}-p \beta / 4 \mid \tilde{\tau}\right)}{\theta_{1}^{\prime}(p \beta / 2 \mid \tilde{\tau}) \theta_{1}\left(\pi u / 2 \tilde{\omega}_{1}+p \beta / 4 \mid \tilde{\tau}\right)} .
$$

Similarly

$$
G(z(u))=\frac{\mu M^{2} \tilde{\omega}_{1}^{2} \cos \frac{p \beta}{2}}{4 \pi^{2} \lambda^{2} \sin ^{3} \frac{p \beta}{2}} \cdot \frac{\theta_{1}(p \beta / 2 \mid \tilde{\tau})^{2}}{\theta_{1}^{\prime}(p \beta / 2 \mid \tilde{\tau})^{2}}\left[\wp\left(u+\tilde{\omega}_{1} p \beta / 2 \pi\right)-\wp\left(\tilde{\omega}_{1} p \beta / \pi\right)\right] .
$$


When the Dijkgraaf-Vafa recipe is followed one finds that for each cut there is an associated filling fraction of eigenvalues $N_{j} / N$ which translates to an effective 't Hooft coupling $S_{j}=g_{s} N_{j}$ with $\sum S_{j}=S$. For the massive vacua we note that the filling fractions in each of the $p$ cuts are the same and equal to $N / p=q$

$$
2 \pi i S_{j}=\frac{d h(\tilde{\tau})}{d \tilde{\tau}} ; \quad j=1, \ldots, p
$$

where $h(\tilde{\tau})$ is the same function that we encountered in (3.20) with $\beta \rightarrow p \beta$ :

$$
h(\tilde{\tau})=\frac{\mu M^{2} \cos \frac{p \beta}{2}}{4 \lambda^{2} \sin ^{3} \frac{p \beta}{2}} \cdot \frac{\theta_{1}(p \beta / 2 \mid \tilde{\tau})}{\theta_{1}^{\prime}(p \beta / 2 \mid \tilde{\tau})} .
$$

In addition one also needs the variation in the genus zero free energy upon transporting an eigenvalue from any given cut to infinity. After minor modifications this is also given by the same expressions that were encountered in the one-cut solution for the confining vacuum:

$$
\sum_{j=1}^{p} \frac{\partial \mathcal{F}_{0}}{\partial S_{j}}=\tilde{\tau} \frac{d h(\tilde{\tau})}{d \tilde{\tau}}-h(\tilde{\tau})-2 \ln \lambda \sum_{j=1}^{p} S_{j}+p \frac{\mu M^{2}}{4 \lambda^{2} \sin ^{2} \frac{\beta}{2}} .
$$

Then the superpotential for the $\mathrm{U}(N)$ theory at any of its massive vacua is

$$
W_{\mathrm{eff}}^{\mathrm{U}(N)}=\frac{N}{p} \sum_{j=1}^{p} \frac{\partial \mathcal{F}_{0}}{\partial S_{j}}-2 \pi i \tau \sum_{j=1}^{p} S_{j}
$$

Extremizing with respect to $\tilde{\tau}$ gives

$$
\tilde{\tau}=\frac{p^{2}}{N} \tau_{R}
$$

and therefore we find that the value of the quantum effective superpotential for the $\mathrm{U}(N)$ theory is

$$
W_{\mathrm{eff}}^{\mathrm{U}(N)}=-\frac{N \mu M^{2} \cos \frac{p \beta}{2}}{4 p \lambda^{2} \sin ^{3} \frac{p \beta}{2}} \cdot \frac{\theta_{1}\left(p \beta / 2 \mid p^{2} \tau_{R} / N\right)}{\theta_{1}^{\prime}\left(p \beta / 2 \mid p^{2} \tau_{R} / N\right)}+\frac{N \mu M^{2}}{4 \lambda^{2} \sin ^{2} \frac{\beta}{2}} .
$$

In all of the above we should remember that $p$ is a divisor of $N$.

The result for $\mathrm{SU}(N)$ follows by the application of Eq. (2.9):

$$
W_{\mathrm{eff}}^{\mathrm{SU}(N)}=\frac{p N \mu M^{2}}{2 \lambda^{2} \sin \beta} \cdot \frac{\theta_{1}^{\prime}\left(p \beta / 2 \mid p^{2} \tau_{R} / N\right)}{\theta_{1}\left(p \beta / 2 \mid p^{2} \tau_{R} / N\right)}-\frac{N \mu M^{2}}{4 \lambda^{2} \sin ^{2} \frac{\beta}{2}} .
$$

This gives the result in only one of $q=N / p$ vacua of this type. The results in the remaining vacua follow by the replacement

$$
\tau_{R} \longrightarrow \tau_{R}+k / p ; \quad k=0,1, \ldots q-1
$$


The Higgs vacuum corresponds to $p=N$ whilst the confining vacuum considered previously has $p=1$ and $k=0$.

One interesting and immediate check on the form of the superpotential follows from the fact that it is manifestly divergent when the deformation parameter takes the special values

$$
\beta=\frac{2 \pi k}{p}, \quad k \in \mathbb{Z} .
$$

But this matches precisely the values of $\beta$ in Eq. (4.4) for which the classical eigenvalues of $\hat{\Phi}$ for the massive vacua diverge (since they consist of $q$ blocks of size $n=p$ ). Another check follows by taking the classical limit of the superpotential (5.14). Using (A.3C), one finds

$$
W_{\mathrm{eff}}^{\mathrm{SU}(N)} \stackrel{\tau_{R} \rightarrow i \infty}{\longrightarrow}\left\langle\operatorname{Tr} \hat{\Phi}^{2}\right\rangle=\frac{N \mu M^{2}}{4 \lambda^{2} \sin ^{2} \frac{\beta}{2}}\left(p \frac{\tan \frac{\beta}{2}}{\tan \frac{p \beta}{2}}-1\right),
$$

which matches the values of $\mu\left\langle\operatorname{Tr} \hat{\Phi}^{2}\right\rangle$ obtained in (4.5) from the $F$-flatness condition precisely.

Now that we have the superpotential in each of the massive vacua, we can now investigate how the full $\mathrm{SL}(2, \mathbb{Z})$ duality group acts on the theory. In the $\mathcal{N}=$ $1^{*}$ theory described in $[7,8]$, we established that the matrix model yields a result for the superpotential which is only duality covariant when shifted by a vacuum independent constant. This kind of additive ambiguity is ubiquitous in this subject: see $[7,9]$. Naturally, the same kind of ambiguity will be present in the Leigh-Strassler deformation where we claim that the appropriate definition of a duality covariant superpotential is

$$
\tilde{W}_{\mathrm{eff}}^{\mathrm{SU}(N)}=W_{\mathrm{eff}}^{\mathrm{SU}(N)}+C\left(\tau_{R}, \beta, \lambda\right),
$$

where the vacuum-independent constant is given by either of the expressions

$$
\begin{aligned}
C\left(\tau_{R}, \beta, \lambda\right) & =\frac{N \mu M^{2}}{4 \lambda^{2} \sin ^{2} \frac{\beta}{2}}-\frac{N \mu M^{2}}{2 \lambda^{2} \sin \beta} \cdot \frac{\theta_{1}^{\prime}\left(\beta / 2 \mid \tau_{R}\right)}{\theta_{1}\left(\beta / 2 \mid \tau_{R}\right)}+\frac{N(N-1) \mu M^{2} \beta}{12 \lambda^{2} \sin \beta} E_{2}\left(\tau_{R}\right) \\
& =\frac{N \mu M^{2}}{4 \lambda^{2} \sin ^{2} \frac{\beta}{2}}-\frac{N \mu M^{2} \omega_{1}}{\pi \lambda^{2} \sin \beta}\left(\zeta\left(\omega_{1} \beta / \pi\right)-\frac{N \beta}{\pi} \zeta\left(\omega_{1}\right)\right) .
\end{aligned}
$$

Here, the Weierstrass $\zeta$-function is defined relative to a torus with periods $\omega_{1}$ and $\omega_{2}$ and $\tau_{R}=\omega_{2} / \omega_{1}$ and $E_{2}\left(\tau_{R}\right)$ is the $2^{\text {nd }}$ Eisenstein series (see the Appendix of [7] for definitions and references). One property that the $C$ has is that it reduces to the correct form needed in the $\mathcal{N}=1^{*}$ theory in the limit $\beta \rightarrow 0$. 
Our claim is that the duality-covariant definition of the superpotential in the each of the massive vacua labelled by $(p, k), p \cdot q=N$ and $k=0, \ldots, q$, is then

$$
\tilde{W}_{\mathrm{eff}}^{\mathrm{SU}(N)}=\frac{N \mu M^{2}}{2 \lambda^{2} \sin \beta}\left(p \frac{\theta_{1}^{\prime}(p \beta / 2 \mid \tilde{\tau})}{\theta_{1}(p \beta / 2 \mid \tilde{\tau})}-\frac{\theta_{1}^{\prime}\left(\beta / 2 \mid \tau_{R}\right)}{\theta_{1}\left(\beta / 2 \mid \tau_{R}\right)}+\frac{\beta}{6}(N-1) E_{2}\left(\tau_{R}\right)\right),
$$

where

$$
\tilde{\tau}=\left(p \tau_{R}+k\right) / q
$$

Using the modular properties of the theta functions and the $2^{\text {nd }}$ Eisenstein series, (A.2a) and (A.5), one can easily show, for example, that

$$
\left.\tilde{W}_{\text {eff }}\left(\beta / \tau_{R},-1 / \tau_{R}, \lambda\right)\right|_{\text {Conf }}=\left.\tau_{R} \frac{\sin \beta}{\sin \left(\beta / \tau_{R}\right)} \cdot \tilde{W}_{\text {eff }}\left(\beta, \tau_{R}, \lambda\right)\right|_{\text {Higgs }} .
$$

Notice that the coupling $\beta$ transforms with unit weight under the modular group. Note also the rather unconventional transformation of the superpotential itself: this we will have to cure by an appropriate definition of the transformation of the coupling $\lambda$.

A consideration of the other massive vacua show that they all lie on a single orbit of the $\operatorname{SL}(2, \mathbb{Z})$ duality group. For the element

$$
\sigma=\left(\begin{array}{ll}
a & b \\
c & d
\end{array}\right) \in \mathrm{SL}(2, \mathbb{Z})
$$

which relates two vacua $A=\sigma(B)$, we have ${ }^{9}$

$$
\left.\tilde{W}_{\text {eff }}\left(\frac{\beta}{c \tau_{R}+d}, \frac{a \tau_{R}+b}{c \tau_{R}+d}, \lambda\right)\right|_{A}=\left.\left(c \tau_{R}+d\right) \frac{\sin \beta}{\sin \left[\beta /\left(c \tau_{R}+d\right)\right]} \cdot \tilde{W}_{\text {eff }}\left(\beta, \tau_{R}, \lambda\right)\right|_{B} .
$$

From this we can deduce the action of $\sigma \in \mathrm{SL}(2, \mathbb{Z})$ on the bare couplings of the theory; clearly

$$
\sigma\left(\tau_{R}\right)=\frac{a \tau_{R}+b}{c \tau_{R}+d}, \quad \sigma(\beta)=\frac{\beta}{c \tau_{R}+d},
$$

confirming that $\beta$ transforms with modular weight -1 . However, we can say more: in order that the superpotential transforms with a definite modular weight, so that it is consistent with the group action, we can deduce the transformation of the coupling $\lambda$. Recall, that on the marginal surface of the Leigh-Strassler deformation $\lambda$ is a function - albeit unknown - of the other two couplings and so must transform. In addition, since in the limit $\beta \rightarrow 0$, the superpotential transforms with modular weight 2 , we expect this to be preserved for $\beta \neq 0$. This requires the combination $\lambda^{2} \sin \beta$ that appears as an overall factor in the superpotential must transform with unit modular weight:

$$
\sigma\left(\lambda^{2} \sin \beta\right)=\frac{\lambda^{2} \sin \beta}{c \tau_{R}+d} .
$$

\footnotetext{
${ }^{9}$ The actual action of $\sigma$ on a vacuum with labels $(p, k)$ is not difficult to elucidate.
} 


\section{An exact elliptic superpotential}

Another method that has been used to calculate the exact values of the superpotential in all the vacua of the $\mathcal{N}=1^{*}$ theory is to compactify it on a circle of finite radius [9]. The effective superpotential is then a function of the dual photons and Wilson lines of the abelian subgroup $\mathrm{U}(1)^{N-1} \subset \mathrm{SU}(N)$. These comprise $N-1$ complex scalar fields $X_{a}, a=\ldots, N$ (with $\sum_{a=1}^{N} X_{a}=0$ ) which naturally live on a torus of complex structure $\tau$ because of the periodicity of each dual photon and Wilson line. The superpotential describing the $\mathcal{N}=1^{*}$ deformation is therefore constrained to be an elliptic function of the complex scalars $X_{a}$. In [9] the exact answer was found to be

$$
W_{\mathbb{R}^{3} \times S^{1}} \sim \sum_{a \neq b} \wp\left(X_{a}-X_{b}\right)
$$

where $\wp(z)$ is the Weierstrass function defined on a torus with half periods $\omega_{1,2}$ with $\tau=\omega_{2} / \omega_{1}$. What is particularly useful about this superpotential is that it is independent of the compactification radius, and therefore, yields results that are valid in the four-dimensional limit, and it encodes all the vacua of the $\mathcal{N}=1^{*}$ theory: both massive and massless.

The question is whether there is an elliptic superpotential that describes the Leigh-Strassler deformation? Similar arguments to those used in [9] suggest that there should be, but are not powerful enough to determine the exact answer. However, there is an obvious candidate for appropriate marginal deformation of (6.1), which turns out to be correct. Specifically, we can replace the Weierstrass function $\wp(z)$, by its unique deformation in the space of even elliptic functions. ${ }^{10}$ In particular, the Weierstrass function has one double pole at the origin and we can consider the elliptic function of order two obtained by splitting the double pole into two single poles. Remarkably, a superpotential of precisely this form reproduces our results from the matrix model for the superpotential in each of the massive vacua. This superpotential can be written as,

$$
W_{\mathbb{R}^{3} \times S^{1}}=\frac{M^{2} \mu}{2 \lambda^{2} \sin \beta}\left(\frac{\omega_{1}}{\pi}\right) \sum_{a \neq b}\left(\zeta\left(X_{a}-X_{b}+\beta \omega_{1} / \pi\right)-\zeta\left(X_{a}-X_{b}-\beta \omega_{1} / \pi\right)\right) .
$$

The Weierstrass-zeta function $\zeta(u)$ can be defined via $\wp(u)=-\zeta^{\prime}(u)$ where $\wp(u)$ is the Weierstrass function for the torus with complex structure $\tau_{R}$. Then in the limit $\beta=0$ this reproduces (6.1). What is remarkable is that the massive vacua of the $\mathcal{N}=1^{*}$ theory for which the $X_{a}$ lie on sub-lattices are also critical points of 6.2 ) since this only relies on the fact that the two-body interaction is an even elliptic

\footnotetext{
${ }^{10}$ The function must be even to respect the Weyl group of $\mathrm{SU}(N)$
} 
function. For instance, for the $p \cdot q=N$ massive vacuum with $k=0$ we have

$$
X_{a} \in\left\{\frac{2 \omega_{1} r}{p}+\frac{2 \omega_{2} s}{q}, \quad r=0, \ldots, p-1, \quad s=0, \ldots, q-1\right\} .
$$

Furthermore, the values of the superpotential are precisely given by (5.20).

A very strong check on the form of the superpotential we have conjectured in (6.2) is possible because of the existence of new vacua which are not present in the limit $\beta=0$. For example, it is easy to show that $X_{a}=0, \forall a$, is a critical point. Actually this is an $\mathrm{SL}(2, \mathbb{Z})$-invariant vacuum. Now we can take the classical limit, $\tau \rightarrow i \infty$, after having shifted by minus the vacuum-independent shift (5.19), and compare with the analysis of the vacuum structure in Section 4 . In the case of SU(3), the classical limit of the superpotential $W_{\mathbb{R}^{3} \times S^{1}}-C$ in the vacuum with $X_{a}=0$ precisely equals the value calculated from the new vacuum solution of the $F$-flatness conditions (4.9). We expect this to generalize to $\mathrm{SU}(N)$, however, the analysis remains to be done.

The properties and construction of this elliptic superpotential will be described in a separate publication; however, we mention the fact that for $\mathcal{N}=1^{*}$, the elliptic superpotential is, more or less, the (complexified) Hamiltonian of the elliptic Calogero-Moser integrable system which described $N$ particles interacting though pair-wise forces proportion to $\wp\left(X_{a}-X_{b}\right)$. The intriguing question is whether this system can be deformed

$$
\wp\left(X_{a}-X_{b}\right) \rightarrow \zeta\left(X_{a}-X_{b}+\beta \omega_{1} / \pi\right)-\zeta\left(X_{a}-X_{b}-\beta \omega_{1} / \pi\right)
$$

whilst preserving integrability.

Finally we comment on a remarkable correspondence between our results for the Leigh-Strassler deformation and the exact superpotential of a certain relevant deformation of the $A_{1}$-quiver theory studied in [14]. Specifically this is the theory of an $\mathrm{SU}(N) \times \mathrm{SU}(N)$ gauge multiplet with gauge couplings $g_{1}$ and $g_{2}$, with two chiral multiplets $A$ and $B$ in the $(N, \bar{N})$ representation and two chiral multiplets $\tilde{A}$ and $\tilde{B}$ in the $(\bar{N}, N)$. The theory also contains two chiral multiplets $\hat{\Phi}_{1}$ and $\hat{\Phi}_{2}$ in the $(\operatorname{adj}, 0)$ and $(0, \operatorname{adj})$ representations respectively with classical superpotential

$$
W=\operatorname{Tr}\left\{\left(g_{1} \hat{\Phi}_{1}+M / 2\right)(A \tilde{A}+B \tilde{B})+\left(g_{2} \hat{\Phi}_{2}+M / 2\right)(\tilde{A} A+\tilde{B} B)\right\} .
$$

This theory actually has $\mathcal{N}=2$ supersymmetry but we further deform it by adding the operator,

$$
\mu \operatorname{Tr}\left(\hat{\Phi}_{1}^{2}-\hat{\Phi}_{2}^{2}\right)
$$

to the classical superpotential which preserves $\mathcal{N}=1$ supersymmetry. This theory is a relevant deformation of the $\mathcal{N}=2$ superconformal theory obtained by setting 
$\mu=M=0$. The exact vacuum structure and superpotential was determined in [14]. The theory has $N \sum_{d \mid N} d$ massive vacua. Remarkably, in $\sum_{d \mid N} d$ of these vacua, we find exactly the same value of the superpotential as in each of the massive vacua of the $\mathrm{SU}(N)$ Leigh-Strassler theory studied in this paper. Specifically, this agreement holds if we identify the parameter $\beta$ of the Leigh-Strassler deformation and the complexified coupling of one of the $\mathrm{SU}(N)$ factors in the quiver theory $\tau_{1}=4 \pi i / g_{1}^{2}+\theta_{1} / 2 \pi$ (or $z=2 i \pi \tau_{1}$ to use the notation of [14]) :

$$
z=\frac{N \omega_{1} \beta}{\pi} .
$$

In the brane set-up for the quiver model $z$ is the relative separation of the two NS5branes. We also identify the parameters $M$ and $\mu$ of the quiver superpotential with parameters of the same name in the deformed Leigh-Strassler superpotential (1.1).

This connection suggests that there should be an RG flow from the superconformal $A_{1}$-quiver to the Leigh-Strassler fixed line. At least in one special case, such a flow is known to exist. In [23], Klebanov and Witten considered the quiver theory in the case of equal gauge couplings $g_{1}=g_{2}$ and zero masses for the bi-fundamental multiplets, $M=0$. In this case one can integrate out the adjoint chiral fields $\hat{\Phi}_{1}$ and $\hat{\Phi}_{2}$ and induce a marginal quartic superpotential for the bi-fundamentals which corresponds to D3-branes placed at a resolved conifold singularity. Moving onto the Higgs branch where $\mathrm{SU}(N) \times \mathrm{SU}(N)$ is broken to the diagonal $\mathrm{SU}(N)$, we find an effective theory of three chiral superfields transforming in the adjoint of the unbroken $\mathrm{SU}(N)$ which coincides with $\mathcal{N}=4$ SUSY Yang-Mills. Repeating this exercise with unequal gauge couplings $g_{1} \neq g_{2}$, we find that the Leigh-Strassler marginal deformation as well as other irrelevant operators are induced. However, it is not obvious whether this flow is related to the exact agreement we have found above.

Acknowledgements: TJH would like to thank Frank Ferrari for discussions. The authors would also like to thank Robbert Dijkgraaf and Cumrun Vafa for valuable discussions. We thank Robbert Dijkgraaf for his comments and observations on a preliminary version of this article. SPK acknowledges support from a PPARC Advanced Fellowship.

\section{Appendix A: Some properties of elliptic functions}

The (quasi-)elliptic functions that we need, $\wp(z), \zeta(z)$ and $\theta_{i}(z \mid \tau)$, are all standard and their definitions and properties can be found in [20]. In our conventions, 
the basic torus has periods $2 \omega_{1}$ and $2 \omega_{2}$ with complex structure $\tau=\omega_{2} / \omega_{1}$. We also define $q=\exp (i \pi \tau)$. Below we list some properties of these functions that will be useful.

An important relation between the Weierstrass and Jacobi theta functions is

$$
\zeta(u)-\frac{\zeta\left(\omega_{1}\right)}{\omega_{1}} u=\frac{\pi}{2 \omega_{1}} \frac{\theta_{1}^{\prime}\left(\pi u /\left(2 \omega_{1}\right) \mid \tau\right)}{\theta_{1}\left(\pi u /\left(2 \omega_{1}\right) \mid \tau\right)} .
$$

The Jacobi theta functions have the following modular properties that will be useful

$$
\begin{aligned}
& \theta_{1}(x \mid \tau)=i(-i \tau)^{-\frac{1}{2}} \exp \left(-i x^{2} / \pi \tau\right) \theta_{1}(x / \tau \mid-1 / \tau) \\
& \theta_{3}(x \mid \tau)=(-i \tau)^{-\frac{1}{2}} \exp \left(-i x^{2} / \pi \tau\right) \theta_{3}(x / \tau \mid-1 / \tau)
\end{aligned}
$$

In order to take the classical limit, we will need the expansion

$$
\begin{aligned}
& \theta_{1}(x \mid \tau)=2 \sum_{n=0}^{\infty}(-1)^{n} q^{(n+1 / 2)^{2}} \sin (2 n+1) x, \\
& \theta_{3}(x \mid \tau)=1+2 \sum_{n=0}^{\infty}(-1)^{n} q^{n^{2}} \cos 2 n x \\
& \frac{\theta_{1}^{\prime}(x \mid \tau)}{\theta_{1}(x \mid \tau)}=\cot x+4 \sum_{n=1}^{\infty} \frac{q^{2 n}}{1-q^{2 n}} \sin 2 n x .
\end{aligned}
$$

We also need the $2^{\text {nd }}$ Eisenstein series [22]

$$
E_{2}(\tau)=1-24 \sum_{n=1} \sigma_{1}(n) q^{2 n}
$$

where $\sigma_{1}(n)$ is a sum over each positive integral divisor of $n$. It has the following non-trivial modular transformation

$$
E_{2}(-1 / \tau)=\tau^{2} E_{2}(\tau)+\frac{6 \tau}{\pi i}
$$

\section{Appendix B: Calculating condensates}

In this appendix we show how the integrals of the type (3.25a) and (3.25b) can be computed to yield the condensates in the $\mathrm{U}(N)$ and $\mathrm{SU}(N)$ gauge theories. Let us first consider the function $f(x)$ in terms of which the eigenvalues have been defined

$$
f(x)=\frac{\theta_{4}(\pi x-\beta / 4 \mid \tilde{\tau})}{\theta_{4}(\pi x+\beta / 4 \mid \tilde{\tau})} \equiv \frac{\theta_{3}(\pi x-\pi / 2-\beta / 4 \mid \tilde{\tau})}{\theta_{3}(\pi x-\pi / 2+\beta / 4 \mid \tilde{\tau})} ; \quad x \in[0,1] .
$$


Clearly, the eigenvalues can be written either in terms of $\theta_{3}$ or $\theta_{4}$ since the only difference is in the range of the argument $x$. We also define the function $g(x)$

$$
g(x)=\frac{\theta_{1}(\pi x-\beta / 4 \mid \tilde{\tau})}{\theta_{1}(\pi x+\beta / 4 \mid \tilde{\tau})} .
$$

The functions $f$ and $g$ have some nice properties

$$
g(x+1)=g(x) ; \quad g(x+\tilde{\tau} / 2)=e^{-i \beta / 2} f(x) ; \quad g(x-\tilde{\tau} / 2)=e^{i \beta / 2} f(x),
$$

which follow from standard identities. Note also that the function $g(x)$ has a simple pole in its fundamental period at $x=1-\beta / 4 \pi$. Now it is a simple matter to convince oneself that because of the periodicity properties of $g(x)$ the contour integral around this pole and hence the residue there can also be written as

$$
\left.\operatorname{Res} g(x)\right|_{x=-\beta / 4 \pi}=-2 i \sin \frac{\beta}{2} \int_{0}^{1} f(x) d x \text {. }
$$

Thus we see that

$$
\int_{0}^{1} f(x) d x=\frac{1}{\sin \frac{\beta}{2}} \frac{\theta_{1}(\beta / 2 \mid \tilde{\tau})}{\theta_{1}^{\prime}(0 \mid \tilde{\tau})} .
$$

From this key result it follows using Eq. (3.24)

$$
\langle\operatorname{Tr} \Phi\rangle=N \int_{0}^{1} d x \phi(x)=\frac{N M}{2 \lambda \sin \frac{\beta}{2}}\left[-\frac{1}{\tan \frac{\beta}{2}} \frac{\theta_{1}(\beta / 2 \mid \tilde{\tau})}{\theta_{1}^{\prime}(\beta / 2 \mid \tilde{\tau})}+1\right]=\frac{2 \lambda \sin \frac{\beta}{2}}{M \mu} W_{\mathrm{eff}}^{\mathrm{U}(N)}
$$

(Note that because $\phi(x)$ is a periodic function under $x \rightarrow x+1$ the actual limits of integration can always be changed from $(-1 / 2,1 / 2)$ to $(0,1)$.) Similarly it also follows that in the $\mathrm{SU}(N)$ theory

$$
\langle\operatorname{Tr} \hat{\Phi}\rangle=N \int_{0}^{1} \hat{\phi}(x) d x=0
$$

To compute the quadratic condensates we need the integrals $\int_{0}^{1} f^{2}(x) d x$. These can also be calculated using the same trick as above and we find that

$$
\int_{0}^{1} f^{2}(x) d x=-\left.\frac{1}{2 i \sin \beta} \operatorname{Res} g^{2}(x)\right|_{x=-\beta / 4 \pi}=\frac{2 \theta_{1}^{\prime}(\beta / 2 \mid \tilde{\tau}) \theta_{1}(\beta / 2 \mid \tilde{\tau})}{\sin \beta \theta_{1}^{\prime}(0 \mid \tilde{\tau})^{2}}
$$

Using the results Eqs. $(\overline{B .5})$ and $(\overline{B .8})$ we can readily compute the quadratic condensates. For the $\mathrm{U}(N)$ theory we find

$$
\left\langle\operatorname{Tr} \Phi^{2}\right\rangle=N \int_{0}^{1} \phi^{2}(x) d x=-\frac{N M^{2}}{2 \lambda^{2} \sin \beta} \cdot \frac{\theta_{1}(\beta / 2 \mid \tilde{\tau})}{\theta_{1}^{\prime}(\beta / 2 \mid \tilde{\tau})}+\frac{N M^{2}}{4 \lambda^{2} \sin ^{2} \frac{\beta}{2}}=\frac{1}{\mu} W_{\mathrm{eff}}^{\mathrm{U}(N)} .
$$


For the $\mathrm{SU}(N)$ theory we find similarly

$$
\left\langle\operatorname{Tr} \hat{\Phi}^{2}\right\rangle=N \int_{0}^{1} \hat{\phi}^{2}(x) d x=\frac{N M^{2}}{2 \lambda^{2} \sin \beta} \cdot \frac{\theta_{1}^{\prime}(\beta / 2 \mid \tilde{\tau})}{\theta_{1}(\beta / 2 \mid \tilde{\tau})}-\frac{N M^{2}}{4 \lambda^{2} \sin ^{2} \frac{\beta}{2}}=\frac{1}{\mu} W_{\mathrm{eff}}^{\mathrm{SU}(N)}
$$

Similarly higher condensates could be computed explicitly if required and will in general depend on the residue of $g^{n}(x)$ at the pole $x=-\beta / 4 \pi$

\section{References}

[1] R. G. Leigh and M. J. Strassler, Nucl. Phys. B 447, 95 (1995) [arXiv:hep-th/9503121].

[2] D. Berenstein, V. Jejjala and R. G. Leigh, Nucl. Phys. B 589, 196 (2000) [arXiv:hep-th/0005087].

[3] D. Berenstein, "Quantum moduli spaces from matrix models," [arXiv:hep-th/0210183].

[4] H. Fuji and Y. Ookouchi, [arXiv:hep-th/0210148].

[5] F. Ferrari, "On exact superpotentials in confining vacua," [arXiv:hep-th/0210135].

[6] R. Dijkgraaf and C. Vafa, "A perturbative window into non-perturbative physics," arXiv:hep-th/0208048; "On geometry and matrix models", arXiv:hep-th/0207106; "Matrix models, topological strings, and supersymmetric gauge theories", arXiv:hep-th/0206255

[7] N. Dorey, T. J. Hollowood, S. Prem Kumar and A. Sinkovics, "Exact superpotentials from matrix models," arXiv:hep-th/0209089.

[8] N. Dorey, T. J. Hollowood, S. P. Kumar and A. Sinkovics, "Massive vacua of N = 1* theory and S-duality from matrix models," arXiv:hep-th/0209099.

[9] N. Dorey, JHEP 9907, 021 (1999) [arXiv:hep-th/9906011].

[10] C. Vafa and E. Witten, Nucl. Phys. B 431, 3 (1994) [arXiv:hep-th/9408074].

[11] N. Dorey and S. P. Kumar, JHEP 0002, 006 (2000) [arXiv:hep-th/0001103].

[12] K. A. Intriligator, Nucl. Phys. B 551 (1999) 575 [arXiv:hep-th/9811047].

[13] O. Aharony, N. Dorey and S. P. Kumar, JHEP 0006 (2000) 026 [arXiv:hep-th/0006008].

[14] N. Dorey, T. J. Hollowood and S. Prem Kumar, Nucl. Phys. B 624, 95 (2002) [arXiv: hep-th/0108221],

T. J. Hollowood and S. P. Kumar, "World sheet instantons via the Myers effect and $\mathrm{N}=1^{*}$ quiver superpotentials," arXiv:hep-th/0206051. 
[15] I. K. Kostov, Nucl. Phys. B 575, 513 (2000) [arXiv:hep-th/9911023].

[16] O. Aharony and S. S. Razamat, JHEP 0205, 029 (2002) [arXiv:hep-th/0204045].

[17] E. Witten, Adv. Theor. Math. Phys. 2, 253 (1998) [arXiv:hep-th/9802150].

[18] J. Polchinski and M. J. Strassler, "The string dual of a confining four-dimensional gauge theory," [arXiv:hep-th/0003136].

[19] S. Katz, P. Mayr and C. Vafa, Adv. Theor. Math. Phys. 1, 53 (1998) [arXiv:hep-th/9706110]; A. E. Lawrence, N. Nekrasov and C. Vafa, Nucl. Phys. B 533, 199 (1998) [arXiv:hep-th/9803015].

[20] E. T. Whittaker, G. N. Watson, A course of Modern Analysis, 4th edn., Cambridge University Press, 1927.

[21] N. Dorey and A. Sinkovics, JHEP 0207, 032 (2002) [arXiv:hep-th/0205151].

[22] N. Koblitz, "Introduction to Elliptic Curves and Modular Forms". Springer-Verlag, 2nd Edition 1993.

[23] I. R. Klebanov and E. Witten, Nucl. Phys. B $536 \quad$ (1998) 199 [arXiv:hep-th/9807080]. 